\title{
Papers
}

\section{Theory and Applications of Four-Wave Mixing in Photorefractive Media}

\author{
MARK CRONIN-GOLOMB, BARUCH FISCHER, JEFFREY O. WHITE, AND AMNON YARIV, FELLOW, IEEE
}

(Invited Paper)

\begin{abstract}
The development of a theory of four-wave mixing in photorefractive crystals is described. This theory is solved in the undepleted pumps approximation with linear absorption and without using the undepleted pumps approximation for negligible absorption. Both the transmission and reflection gratings are treated individually. The results are used to analyze several photorefractive phase conjugate mirrors, yielding reflectivities and thresholds. The use of photorefractive crystals as optical distortion correction elements and experimental demonstrations of several of the passive phase conjugate mirrors are described.
\end{abstract}

\section{INTRODUCTION}

$\mathrm{T}$ HROUGH the recent years' boom in nonlinear optical phase conjugation, photorefractive (PR) materials such as $\mathrm{LiNbO}_{3}, \mathrm{Bi}_{12} \mathrm{Si}_{20} \mathrm{O}_{3}$ (BSO), $\mathrm{BaTiO}_{3}$, and $\mathrm{Sr}_{1-x} \mathrm{Ba}_{x} \mathrm{Nb}_{2} \mathrm{O}_{6}$ (SBN) have been assuming ever increasing prominence due to their unique capability for displaying strong nonlinear effects with milliwatt beams over the entire visible spectrum and beyond.

In the late 1960's it was noticed that certain frequency doubling crystals were subject to "optical damage" characterized by degraded phase matching due to light induced changes in refractive index [1]-[3]. These index variations persisted in the dark, sometimes for many months, and could be erased by flooding the crystal with uniform illumination. That the effect could actually be put to use was noticed by those interested in real-time volume holography who thought to use PR materials as very dense optical memories $\left(\approx 10^{11}\right.$ bits $\left./ \mathrm{cm}^{3}\right)$ and for real-time optical information processing [4]-[21]. Concurrent theoretical developments involved both investigation of a) the physical mechanism whereby light intensity was converted to refractive index variations and $b$ ) the optical nonlinearities involved in the beam coupling due to the selfdeveloping hologram [22]-[34]. While no true third-order constitutive nonlinearity was involved, the diffraction of two beams into each other by the very grating they wrote resulted in strong third-order interactions. The physics of the PR effect is such that the index grating is often $\pi / 2$ out of phase in space with the interference pattern and one beam is coherently amplified at the expense of the other. The implications for coherent optical processing are clear.

Manuscript received June 27, 1983; revised September 7, 1983. This work was supported by the U.S. Air Force Office of Scientific Research, the U.S. Army Research Office, Durham, NC, and Rockwell International.

M. Cronin-Golomb, J, O. White, and A. Yariv are with the California Institute of Technology, Pasadena, CA 91125.

B. Fischer is with the Department of Electrical Engineering, Technion, Haifa, Israel.
In the mid 1970's the field of phase conjugate optics [35] began to prosper because of potential applications in aberration correction. The main problem to be faced was and still is the development of fast media with large nonlinearities. While phase conjugation by four-wave mixing is often thought of in terms of nonlinear optical susceptibilities, the formal analogy with real-time holography [36] has made it clear that PR materials could be used effectively in phase conjugation. As a result, a whole new branch in phase conjugate optics has been developed [37]-[40]. High reflectivity phase conjugation with low-power lasers has become an easy task. Feinberg has demonstrated a resonator using a PR phase conjugate mirror [41], and many new nonlinear optical devices have been demonstrated including ring oscillators by White $e t$ al. [42] and passive phase conjugate mirrors (PPCM's) by CroninGolomb et al. [43]-[45], Feinberg [46], and Odulov and Soskin [47].

Among the PR materials that we have used so far, we find $\mathrm{BaTiO}_{3}$ (first used in four-wave mixing by Feinberg et al. [32]) and SBN (first used in four-wave mixing by Fischer et al. [48]) most suited to experiments where large coupling strengths are required since they have very large electrooptic coefficients. The main disadvantage of these materials is their slow response times, typically about $10 / I \mathrm{~s}$ where $I$ is the total intensity of the interacting beams in $\mathrm{mW} / \mathrm{mm}^{2}$. One main research direction in the future is going to be optimization of this time; much work has already been done with $\mathrm{LiNbO}_{3}$ where the use of reduced iron impurities seems to be effective [49].

In this paper we will describe the more recent developments in the use of PR materials in phase conjugation and other applications of nonlinear optics. Section II details the coupling mechanism for two- and four-wave mixing in PR materials. We present the four-coupled wave equations and solve them in the undepleted pumps and single grating approximations. The effects on phase conjugation of the spatial phase shift between the index grating and the light interference pattern are discussed. In Section III we obtain solutions in which no use is made of the undepleted pumps approximation. We describe the multistability that results from considering the full nonlinear nature of the problem. In Section IV we explain the application of the theory to the new PPCM's and show the results of experimental demonstrations of these devices. We also introduce novel interpretations of PR crystals as distortion correcting elements in optical systems and circuits and as double phase conjugate mirrors. Section V is a summary. 


\section{Coupled Wave Equations and Undepleted Pumps APPROXIMATION THEORY}

Microscopic theories of the photorefractive effect generally treat photoionization of charge carriers from impurity levels. These carriers are subject to drift and diffusion in the spatially varying intensity of the recording beams and the electric field associated with the resultant space charge operates through the electrooptic effect to modulate the index of refraction. This effect is nonlocal, with one manifestation being that the index grating is not in phase with the interference pattern. For this reason, phase conjugation by degenerate four-wave mixing in PR crystals is different in kind than phase conjugation in other media and we cannot characterize the medium response by a simple constitutive third-order nonlinear constant $\chi^{(3)}$. The derivation of the coupled wave equations with the associated effective third-order nonlinearities is as follows.

The basic interaction geometry is illustrated in Fig. 1. Four waves of equal frequency $\omega$ and, for simplicity, of the same polarization are propagating through the PR medium. Let the electric field amplitude associated with the $j$ th beam be

$$
E_{j}(r, t)=A_{j}(r) \exp \left[i\left(\boldsymbol{k}_{j} \cdot \boldsymbol{r}-\omega t\right)\right]+\text { c.c. }
$$

We solve the problem in steady state so that the $A_{j}$ may be taken to be time independent. The propagation directions come in two oppositely directed pairs, $\boldsymbol{k}_{1}=-\boldsymbol{k}_{2}$ and $\boldsymbol{k}_{3}=-\boldsymbol{k}_{4}$, whereas the relative direction of $\boldsymbol{k}_{1}$ and $\boldsymbol{k}_{4}$ is arbitrary.

It is the fringes in the time independent part of the light intensity that generate the hologram, whose fringes have the same periodicity as the light interference pattern. In general, the holographic fringes of refractive index will have a spatial phase shift with respect to the interference pattern, so we can write the fundamental components of the intensity induced grating as

$$
\begin{aligned}
n= & n_{0}+\frac{n_{\mathrm{I}} e^{i \varphi_{\mathrm{I}}}}{2} \frac{\left(A_{1}^{*} A_{4}+A_{2} A_{3}^{*}\right)}{I_{0}} \exp \left(i \boldsymbol{k}_{\mathrm{I}} \cdot \boldsymbol{r}\right)+\text { c.c. } \\
& +\frac{n_{\mathrm{II}} e^{i \varphi}}{2} \frac{\left(A_{1} A_{3}^{*}+A_{2}^{*} A_{4}\right)}{I_{0}} \exp \left(i \boldsymbol{k}_{\mathrm{II}} \cdot \boldsymbol{r}\right)+\text { c.c. } \\
& +\frac{n_{\mathrm{III}} e^{i \varphi_{\mathrm{III}}}}{2} \frac{\left(A_{1} A_{2}^{*}\right)}{I_{0}} \exp \left(i \boldsymbol{k}_{\mathrm{III}} \cdot \boldsymbol{r}\right)+\text { c.c. } \\
& +\frac{n_{\mathrm{IV}} e^{i \varphi \mathrm{IV}}}{2} \frac{\left(A_{3}^{*} A_{4}\right)}{I_{0}} \exp \left(i \boldsymbol{k}_{\mathrm{IV}} \cdot \boldsymbol{r}\right)+\text { c.c. }
\end{aligned}
$$

where

$$
I_{0}=\sum_{j=1}^{4} I_{j}
$$

with $I_{j}$ the intensity $\left|A_{j}\right|^{2}$ of beam $j$. Through this normalization by $I_{0}$ we anticipate that the coupling strengths in the PR effect are approximately independent of total intensity, in direct contrast with four-wave mixing via nonlinear polarizabilities. The phases $\varphi_{\mathrm{I}}, \varphi_{\mathrm{II}}, \varphi_{\mathrm{III}}$, and $\varphi_{\mathrm{IV}}$ are real and $n_{\mathrm{I}}, n_{\mathrm{II}}$, $n_{\mathrm{III}}$, and $n_{\mathrm{IV}}$ are real and positive. $k_{\mathrm{I}}=k_{4}-k_{1}=k_{2}-k_{3}$, $k_{\mathrm{II}}=k_{1}-k_{3}=k_{4}-k_{2}, k_{\mathrm{III}}=2 k_{1}$ and $k_{\mathrm{IV}}=2 k_{4}$. The com. plex constant $n_{\mathrm{I}} e^{i \varphi_{\mathrm{I}}}$ as an example, characterizes the spatial hologram written by the intensity interference pattern of

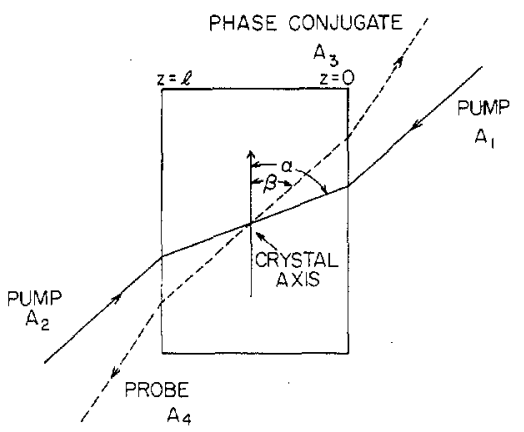

Fig. 1. Four-wave mixing arrangement appropriate to phase conjugation showing the pump beams (solid) and probe and phase conjugate beams (dashed), as well as the relative orientation of the $c$-axis of the crystal.

beams 1 and 4 and also that of beams 2 and 3. These two pairs of waves are characterized by the same constant because $k_{4}-k_{1}=k_{2}-k_{3}$.

The expressions for these various constants are obtained by solving the specific physical process responsible for the hologram formation. Expressions for $n_{\mathrm{I}}$ and $\varphi_{\mathrm{I}}$, for example, derived from a typical rate equation model are [31]

$$
\begin{aligned}
n_{\mathrm{I}} & =-\frac{r_{\mathrm{eff}} n_{0}^{3} E_{p}}{2}\left[\frac{E_{0}^{2}+E_{d}^{2}}{E_{0}^{2}+\left(E_{d}+E_{p}\right)^{2}}\right]^{1 / 2} \\
\tan \varphi_{\mathrm{I}} & =\frac{E_{d}\left(E_{d}+E_{p}\right)+E_{0}^{2}}{E_{0} E_{p}}
\end{aligned}
$$

where $n_{0}$ is the ordinary refractive index, $r_{\text {eff }}$ is the relevant electrooptic coefficient, $E_{0}$ is a superimposed spatially uniform electric field, either applied or intrinsic (due, e.g., to the photovoltaic effect [7]) directed along $\boldsymbol{k}_{\mathrm{I}}$, and $E_{d}$ and $E_{p}$ are electric fields characteristic of diffusion and maximum space charge, respectively. $E_{d}=k_{B} T k_{\mathrm{I}} / \mathrm{e}$ and $E_{p}=\mathrm{e} p_{d} /\left(\epsilon k_{\mathrm{I}}\right)$ where $p_{d}$ is the density of traps in the material, $k_{B}$ is Boltzmann's constant, $T$ is the temperature, $e$ is the electron charge, $\epsilon$ is the permittivity of the material and $k_{\mathrm{I}}=\left|k_{\mathrm{I}}\right|$. The electrooptic coefficient is given by

$$
r_{\text {eff }}=\epsilon_{i i}\left(r_{i j k} k_{\mathrm{I} k}\right) \epsilon_{j j} /\left(k_{\mathrm{I}} n_{0}^{3} n_{\lambda}\right)
$$

where $n_{\lambda}$ is $n_{\mathrm{e}}$ or $n_{0}$ depending on whether the mixing beams are of extraordinary or ordinary polarization. For crystals of the point group $4 \mathrm{~mm}$ such as $\mathrm{SBN}$ and $\mathrm{BaTiO}_{3}$, the nonzero electrooptic coefficients and their conventional contracted notations are $r_{z z z} \equiv r_{33}, r_{x x z}=r_{y y z} \equiv r_{13}$, and $r_{y z y}=r_{x x x} \equiv$ $r_{42}$. Equation (2.6a) reduces to

$$
r_{\text {eff }}=r_{13} \sin [(\alpha+\beta) / 2]
$$

for mixing beams of ordinary polarization and

$$
\begin{aligned}
r_{\mathrm{eff}}= & \left\{n_{\mathrm{e}}^{4} r_{33} \sin \alpha \sin \beta+2 n_{\mathrm{e}}^{2} n_{0}^{2} r_{42} \cos ^{2}[(\alpha+\beta) / 2]\right. \\
& \left.+n_{0}^{4} r_{13} \cos \alpha \cos \beta\right\} \sin [(\alpha+\beta) / 2] /\left(n_{\mathrm{e}} n_{0}^{3}\right)
\end{aligned}
$$

for mixing beams of extraordinary polarization where $\alpha$ and $\beta$ are the angles of the pump beams and the probe and phase conjugate beams with respect to the optic axis of the crystal as shown in Fig. 1. The $r_{i j}$ are the electrooptic coefficients and $n_{0}$ and $n_{\mathrm{e}}$ are the ordinary and extraordinary refractive indexes, respectively.

In $\mathrm{BaTiO}_{3}$ the large electrooptic coefficient [50] is $r_{42}$. To 
observe the largest effects it is necessary to use extraordinary polarization and to orient the crystal so that the grating wave vector is not parallel to any of the crystal axes. In SBN, on the other hand, it is $r_{33}$ that is large [48] $\left(\approx 10^{-9} \mathrm{~m} / \mathrm{V}\right)$. While it is still necessary to use extraordinary polarization, the grating wave vector can here be parallel to the optic axis of the crystal.

Now, by using (2.2) in the wave equation

$$
\nabla^{2} E+k^{2} E=0
$$

we can derive the following four-coupled wave equations by the standard slowly varying field approximation [50], [51] and with $\alpha_{1}=\alpha / \cos \theta_{1}$ and $\alpha_{2}=\alpha / \cos \theta_{2}$ where $\alpha$ is the linear absorption and $\vartheta_{1}$ and $\vartheta_{2}$ are the angles of pump 1 and probe 4 with respect to the normal to the crystal surface.

$$
\begin{aligned}
\frac{2 c}{\omega} \cos \vartheta_{1} \frac{d A_{1}}{d z}= & -\frac{i n_{1} e^{-i \varphi_{\mathrm{I}}}}{I_{0}}\left(A_{1} A_{4}^{*}+A_{2}^{*} A_{3}\right) A_{4} \\
& -\frac{i n_{\mathrm{II}} e^{i \varphi \mathrm{II}}}{I_{0}}\left(A_{1} A_{3}^{*}+A_{2}^{*} A_{4}\right) A_{3} \\
& -\frac{i n_{\mathrm{III}} e^{i \varphi_{\mathrm{III}}}}{I_{0}}\left(A_{1} A_{2}^{*}\right) A_{2} \\
& -\frac{2 c}{\omega} \cos \vartheta_{1} \alpha_{1} A_{1}
\end{aligned}
$$

$$
\begin{aligned}
\frac{2 c}{\omega} \cos \vartheta_{1} \frac{d A_{2}}{d z}= & \frac{i n_{\mathrm{I}} \mathrm{e}^{i \varphi_{\mathrm{I}}}}{I_{0}}\left(A_{1}^{*} A_{4}+A_{2} A_{3}^{*}\right) A_{3} \\
& +\frac{i n_{\mathrm{II}} e^{-i \varphi_{\mathrm{II}}}}{I_{0}}\left(A_{1}^{*} A_{3}+A_{2} A_{4}^{*}\right) A_{4} \\
& +\frac{i n_{\mathrm{II}} e^{-i \varphi \mathrm{III}}}{I_{0}}\left(A_{1}^{*} A_{2}\right) A_{1} \\
& +\frac{2 c}{\omega} \cos \vartheta_{1} \alpha_{1} A_{2}
\end{aligned}
$$

$$
\begin{aligned}
\frac{2 c}{\omega} \cos \vartheta_{2} \frac{d A_{3}}{d z}= & \frac{i n_{\mathrm{I}} e^{-i \varphi_{\mathrm{I}}}}{I_{0}}\left(A_{1} A_{4}^{*}+A_{2}^{*} A_{3}\right) A_{2} \\
& +\frac{i n_{\mathrm{II}} e^{-i \varphi_{\mathrm{II}}}}{I_{0}}\left(A_{1}^{*} A_{3}+A_{2} A_{4}^{*}\right) A_{1} \\
& +\frac{i \eta_{\mathrm{IV}} e^{-i \varphi \mathrm{IV}}}{I_{0}}\left(A_{3} A_{4}^{*}\right) A_{4} \\
& +\frac{2 c}{\omega} \cos \vartheta_{2} \alpha_{2} A_{3}
\end{aligned}
$$

$$
\begin{aligned}
\frac{2 c}{\omega} \cos \vartheta_{2} \frac{d A_{4}}{d z}= & -\frac{i n_{1} e^{i \varphi_{\mathrm{I}}}}{I_{0}}\left(A_{1}^{*} A_{4}+A_{2} A_{3}^{*}\right) A_{1} \\
& -\frac{i n_{\mathrm{II}} e^{i \varphi_{\mathrm{II}}}}{I_{0}}\left(A_{1} A_{3}^{*}+A_{2}^{*} A_{4}\right) A_{2}
\end{aligned}
$$

$$
\begin{aligned}
& -\frac{i n_{\mathrm{IV}} e^{i \varphi_{\mathrm{IV}}}}{I_{0}}\left(A_{3}^{*} A_{4}\right) A_{3} \\
& -\frac{2 c}{\omega} \cos \vartheta_{2} \alpha_{2} A_{4} .
\end{aligned}
$$

When $A_{3}$ and $A_{2}$ are taken to be zero in the above equations, we recover the well-known theory of holographic two-beam coupling [26], [31]. There too, the spatial phase difference $\varphi_{1}$ between the light interference pattern and the index grating plays an important role. Its sign determines the direction of energy transfer from one beam to the other. It introduces an asymmetry that allows one beam to be amplified by constructive interference with radiation scattered by the grating, while the other beam is attenuated by destructive interference with diffracted radiation. In the present analysis of phase conjugation, we will show that this leads to an asymmetry between the effects of the counterpropagating pumping beams 1 and 2.

\section{The Transmission Grating in the Undepleted Pumps Approximation}

The problem may be simplified by making two assumptions. First, we consider a holographic system whose spatial frequency response is such that of all the gratings present in the system, only one gives rise to strong beam coupling. This predominance of one grating is enforced in most practical situations by a choice of the directions, polarization, and coherence relationships of the four beams relative to the crystal axes and to the application in some cases of an electric field that enhances certain gratings. Here, we consider the case of the transmission grating where only $n_{\mathrm{I}}$ is nonzero. In addition, we make the assumption $\vartheta_{1}=\vartheta_{2}$ so that $\alpha_{1}=\alpha_{2}$, and we may drop the subscripts on the $\alpha$ and the $\theta$. The equations reduce to

$$
\begin{aligned}
& \frac{d A_{1}}{d z}=-\frac{\gamma}{I_{0}}\left(A_{1} A_{4}^{*}+A_{2}^{*} A_{3}\right) A_{4}-\alpha A_{1} \\
& \frac{d A_{2}^{*}}{d z}=-\frac{\gamma}{I_{0}}\left(A_{1} A_{4}^{*}+A_{2}^{*} A_{3}\right) A_{3}^{*}+\alpha A_{2}^{*} \\
& \frac{d A_{3}}{d z}=\frac{\gamma}{I_{0}}\left(A_{1} A_{4}^{*}+A_{2}^{*} A_{3}\right) A_{2}+\alpha A_{3} \\
& \frac{d A_{4}^{*}}{d z}=\frac{\gamma}{I_{0}}\left(A_{1} A_{4}^{*}+A_{2}^{*} A_{3}\right) A_{1}^{*}-\alpha A_{4}^{*}
\end{aligned}
$$

where we have defined the coupling constant $\gamma$ by

$$
\gamma=\frac{i \omega n_{\mathrm{I}} e^{-i \varphi_{\mathrm{I}}}}{2 c \cos \vartheta} .
$$

Secondly, in this section, we make use of the undepleted pumps approximation in which $I_{1}, I_{2} \gg I_{3}, I_{4}$. In this case, the nonlinear terms in (2.9a), (2.9b) are of the order of $A_{4}^{2}$ or $A_{3} A_{4}$, and can be neglected. With these approximations and boundary conditions appropriate for phase conjugation, $\left[A_{3}(l)=0\right.$ and $A_{4}^{*}(0)$ known], the solution of (2.9) is 


$$
\begin{aligned}
& A_{1}(z)=A_{1}(0) e^{-\alpha z} \\
& A_{2}(z)=A_{2}(l) e^{\alpha(z-l)} \\
& A_{3}(z)=-\frac{A_{2}(l) A_{4}^{*}(0) e^{\alpha(z-l)+\gamma z}}{A_{1}^{*}(0)} \frac{J(z)}{J(0)-1} \\
& A_{4}^{*}(z)=-A_{4}^{*}(0) e^{-\alpha z+\gamma z}\left[\frac{J(z)-e^{-\gamma z}}{J(0)-1}\right]
\end{aligned}
$$

where

$$
J(z)=\int_{\gamma z}^{\gamma l} \frac{\exp (-x) d x}{1+r e^{-2 \hat{\alpha} \gamma l} \exp (4 \hat{\alpha} x)}
$$

where $r$ is the pump ratio $I_{2}(l) / I_{1}(0)$ and $\hat{\alpha}$ is the normalized linear absorption $\alpha / \gamma$. The phase conjugate reflectivity is thus

$$
\rho \equiv \frac{A_{3}(0)}{A_{4}^{*}(0)}=-\frac{A_{2}(l)}{A_{1}^{*}(0)} \frac{J(0) e^{-\alpha l}}{J(0)-1} .
$$

In Fig. 2 we plot the phase conjugate intensity reflectivity $R=|\rho|^{2}$ as a function of pump ratio for $\gamma l= \pm 3$ and for various values of the normalized linear absorption $\hat{\alpha}$. We see that the effect of increasing linear absorption is primarily to decrease the reflectivity, with the greater decrease being for negative $\gamma l$. We observe too that the reflectivity for zero absorption is unchanged under the transformation $r \rightarrow 1 / r$ and $\gamma \rightarrow-\gamma$. This means that probe beams traveling in opposite directions to each other will experience the same reflectivity from a given PR phase conjugate mirror. This is of considerable practical importance in the design of various optical resonator devices based on four-wave mixing in PR crystals.

The integration for the function $J$ can be explicitly performed when $\hat{\alpha}$ is zero. In that case, the solutions given in (2.11) reduce to

$$
\begin{aligned}
& A_{1}(z)=A_{1}(0) \\
& A_{2}(z)=A_{2}(l) \\
& A_{3}(z)=\frac{A_{2}(l) A_{4}^{*}(0)}{A_{1}^{*}(0)}\left[\frac{e^{\gamma(z-l)}-1}{e^{-\gamma l}+r}\right] \\
& A_{4}^{*}(z)=A_{4}^{*}(0)\left[\frac{e^{\gamma(z-l)}+r}{e^{-\gamma l}+r}\right]
\end{aligned}
$$

so that the reflectivity is simply

$$
\rho=-\left[\frac{A_{1} A_{2}}{A_{1}^{*} A_{2}^{*}}\right]^{1 / 2} \frac{\sinh \left(\frac{1}{2} \gamma l\right)}{\cosh \left(\frac{1}{2} \gamma l+\frac{1}{2} \ln r\right)} .
$$

When the phase shift is zero and a) the magnitude of the coupling strength crystal length product is $\pi$, and $b$ ) the pumping beams are of equal intensity, the reflectivity becomes infinite, corresponding to the self-oscillation effect [52]: we have finite output for zero input. Fig. 3 is a graph of the reflectivity for various phase shifts. In each case the magnitude of the coupling strength length product is the same. The most important feature

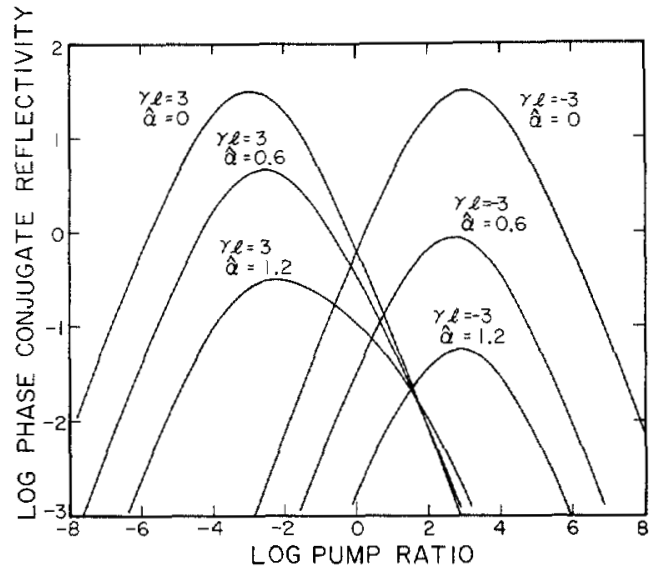

Fig. 2. Phase conjugate intensity reflectivity as a function of pump ratio $I_{2}(l) / I_{1}(0)$ for coupling constant $\gamma l= \pm 3$ and for various values of the linear absorption normalized to $\gamma: \hat{\alpha}=\alpha / \gamma$.

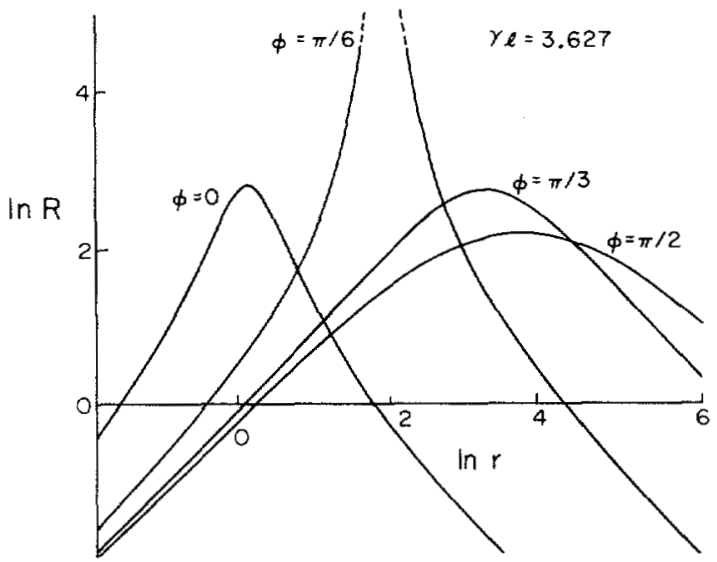

Fig. 3. Phase conjugate reflectivity for coupling strength $\gamma l=-3.627$ and peaking from left to right, $\varphi=0, \pi / 6, \pi / 3$, and $\pi / 2$, Mirrorless self-oscillation occurs here for $\varphi=\pi / 6$ and $r=6.13$.

we observe here is that with the nonzero phase shifts common in PR four-wave mixing the intensities of the pumping beams should be unequal for optimum reflectivity. This is in direct contrast with the situation familiar in four-wave mixing in media with a local response where the intensities of the pumping beams should be equal. Also, with the $90^{\circ}$ phase shift common in our crystals, we find that self-oscillation is not possible. However, by detuning the probe beam from the pump beams, the holographic phase shift is changed since the slowly responding grating lags behind the moving interference fringes. Recently, Lam pointed out that this departure from $90^{\circ}$ phase shift can cause self-oscillation [53]. This is shown here in the graph for phase shift $\pi / 6$. We should also mention that the phase can be adjusted by applying an electric field; in that case, self-oscillation should again become possible.

\section{The Reflection Grating in the Undepleted Pumps Approximation}

The results above were derived with reference to the case $n_{1}$ nonzero where experimental conditions were such that the interference of beams 1 and 4 and beams 2 and 3 induced a transmission grating. If instead, because of changes in the relative coherence of the interacting beams or because of different crystal orientation, it is the interference between beams 1 and 3 and beams 2 and 4 that is effective in hologram writing, then 
a reflection grating will result. This is described in the coupled wave equations ( 2.8 ) by taking $n_{\mathrm{II}}$ nonzero, all other coupling constants being negligible. The applicable coupled wave equations reduce to

$$
\begin{aligned}
& \frac{d A_{1}}{d z}=\frac{\gamma}{I_{0}}\left(A_{1} A_{3}^{*}+A_{2}^{*} A_{4}\right) A_{3}-\alpha A_{1} \\
& \frac{d A_{2}^{*}}{d z}=\frac{\gamma}{I_{0}}\left(A_{1} A_{3}^{*}+A_{2}^{*} A_{4}\right) A_{4}^{*}+\alpha A_{2}^{*} \\
& \frac{d A_{3}^{*}}{d z}=\frac{\gamma}{I_{0}}\left(A_{1} A_{3}^{*}+A_{2}^{*} A_{4}\right) A_{1}^{*}+\alpha A_{3}^{*} \\
& \frac{d A_{4}}{d z}=\frac{\gamma}{I_{0}}\left(A_{1} A_{3}^{*}+A_{2}^{*} A_{4}\right) A_{2}-\alpha A_{4}
\end{aligned}
$$

where the coupling constant $\gamma$ is now given by

$$
\gamma=\frac{-i \omega n_{\mathrm{II}} e^{i \varphi} \mathrm{II}}{2 c \cos \vartheta}
$$

So that we may compare the results for the transmission and reflection gratings more closely, we rename the beams without changing the physics: $A_{1} \rightarrow A_{3} \rightarrow A_{2} \rightarrow A_{4} \rightarrow A_{1}$ (Fig. 4). It will now be easier to look for basic differences and similarities. The only change has been the direction of the crystal surfaces.

$$
\begin{aligned}
& \frac{d A_{1}}{d z}=\frac{\gamma}{I_{0}}\left(A_{1} A_{4}^{*}+A_{2}^{*} A_{3}\right) A_{4}-\alpha A_{1} \\
& \frac{d A_{2}^{*}}{d z}=\frac{\gamma}{I_{0}}\left(A_{1} A_{4}^{*}+A_{2}^{*} A_{3}\right) A_{3}^{*}+\alpha A_{2}^{*} \\
& \frac{d A_{3}}{d z}=\frac{\gamma}{I_{0}}\left(A_{1} A_{4}^{*}+A_{2}^{*} A_{3}\right) A_{2}+\alpha A_{3} \\
& \frac{d A_{4}^{*}}{d z}=\frac{\gamma}{I_{0}}\left(A_{1} A_{4}^{*}+A_{2}^{*} A_{3}\right) A_{1}^{*}-\alpha A_{4}^{*} .
\end{aligned}
$$

The close similarity between the coupled wave equations for the reflection and transmission gratings is readily apparent, The only difference between them is the sign of the nonlinear terms in the pump equations (2.9a), (2.9b) and (2.18a), (2.18b). It follows immediately that the results for the transmission grating and the reflection grating are the same in the undepleted pumps approximation. As we show in the next section, however, the inclusion of pump depletion introduces important differences in the behaviors of the two types of grating.

\section{Exact Solution of the Coupled Wave Equations with Negligible Linear Absorption}

Four-wave mixing coupled wave equations with pump depletion were first solved in 1979 by Marburger and Lam who used a Lagrangian method [54], [55]. Subsequently, graphs of phase conjugate reflectivity much like those shown in Fig. 5 were produced [56]. This solution, however, could not accommodate the photorefractive phase shifts between interference pattern and index grating. It was also only strictly valid for collinear pump and probe. In that case, extra polarization terms arose in the coupled wave equations which spoiled the phase conjugate nature of the reflected signal. Since then, Kessel and Musin have presented a solution for a very general

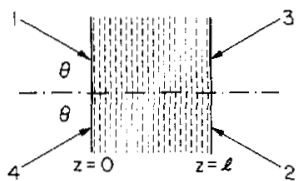

(a)

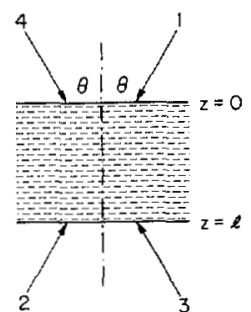

(b)

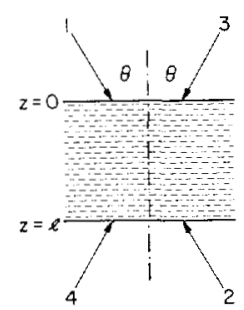

(c)

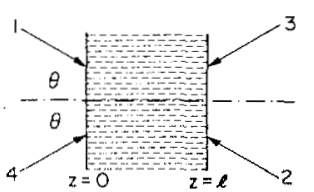

(d)

Fig. 4. A transformation to facilitate comparision between transmission and reflection gratings. (a) The reflection grating. (b) The reflection grating rotated $90^{\circ}$. (c) The rotated reflection grating with renamed beams is now directly comparable to the transmission grating. (d) The transmission grating.

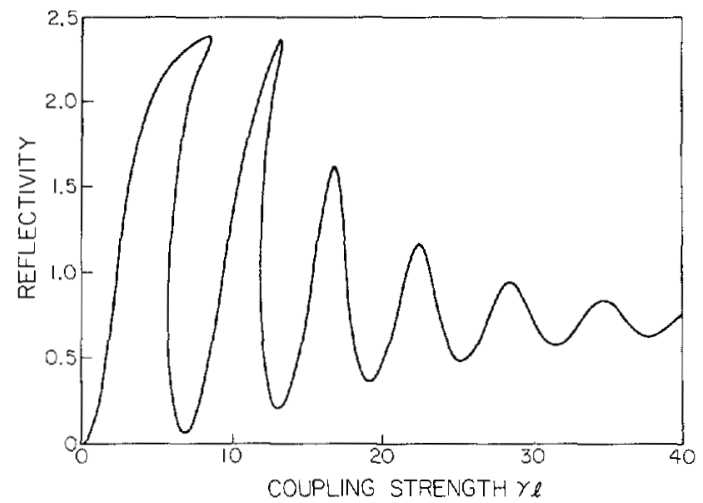

Fig. 5. Reflectivity of a PR phase conjugate mirror versus coupling strength magnitude $|\gamma| \mid$. The incident pump beams are of equal intensity $(r=1)$, the intensity of the incident probe beam is 20 percent of the total incident pumping intensity and the phase shift between index grating and the interference fringes is $5^{\circ}$. The fourwave mixing is via the transmission grating.

class of nonlinear parametric processes including four-wave mixing [57]. This solution does not require the finding of conservation laws for the decoupling of the equations. How ever, it is still only valid for local nonlinear susceptibilities. In the paragraphs below, we find solutions for a system with nonlocal susceptibilities, the photorefractive crystal. It should be pointed out that all of these analyses derive the intensities and not the phases of the various beams. The effects of 
strong nonlinearities on the phases of the output beams are not yet understood, and are the subject of current theoretical efforts. These effects will be important in considerations of the faithfulness of phase conjugation and the performance of resonators employing phase conjugate mirrors.

\section{The Transmission Grating}

We develop here the exact solution of (2.9) for four-wave mixing in PR media by the transmission grating with negligible linear absorption [58]. The first step is to write down a set of conservation laws.

$$
\begin{aligned}
A_{1} A_{2}+A_{3} A_{4} & =c_{1} \equiv c \\
A_{1} A_{3}^{*}-A_{2}^{*} A_{4} & =c_{2} \\
I_{1}+I_{4} & =d_{1} \\
I_{2}+I_{3} & =d_{2}
\end{aligned}
$$

where $c_{1} \equiv c, c_{2}, d_{1}$, and $d_{2}$ are constants of integration. These relations may be checked easily using the coupled wave equations.

With the help of these conservation laws, $(2.9 a)$ and $(2.9 b)$ with zero absorption can be decoupled from (2.9c) and (2.9d).

$$
\begin{aligned}
& \frac{d A_{1}}{d z}=-\frac{\gamma}{I_{0}}\left[A_{1} d_{1}-A_{1}\left(I_{1}+I_{2}\right)+A_{2}^{*} c\right] \\
& \frac{d A_{2}^{*}}{d z}=-\frac{\gamma}{I_{0}}\left[A_{1} c^{*}-A_{2}^{*}\left(I_{1}+I_{2}\right)+A_{2}^{*} d_{2}\right] \\
& \frac{d A_{3}}{d z}=\frac{\gamma}{I_{0}}\left[A_{3} d_{2}-A_{3}\left(I_{3}+I_{4}\right)+A_{4}^{*} c\right] \\
& \frac{d A_{4}^{*}}{d z}=\frac{\gamma}{I_{0}}\left[A_{3} c^{*}-A_{4}^{*}\left(I_{3}+I_{4}\right)+A_{4}^{*} d_{1}\right] .
\end{aligned}
$$

By eliminating the term in $I_{1}+I_{2}$ between (3.2a) and (3.2b), and the term in $I_{3}+I_{4}$ between $(3.2 \mathrm{c})$ and (3.2d), we find the following expressions for $A_{12} \equiv A_{1} / A_{2}^{*}$ and $A_{34} \equiv A_{3} / A_{4}^{*}$.

$$
\begin{aligned}
& \frac{d A_{12}}{d z}=-\frac{\gamma}{I_{0}}\left[c+\left(d_{1}-d_{2}\right) A_{12}-c^{*} A_{12}^{2}\right] \\
& \frac{d A_{34}}{d z}=\frac{\gamma}{I_{0}}\left[c+\left(d_{2}-d_{1}\right) A_{34}-c^{*} A_{34}^{2}\right] .
\end{aligned}
$$

Noting that $I_{0}$ is constant because of the conservation laws, we see that these equations are immediately integrable

$$
\begin{aligned}
& A_{12}(z)=-\left[\frac{S_{-} D e^{-\mu z}-S_{+} D^{-1} e^{\mu z}}{2 c^{*}\left(D e^{-\mu z}-D^{-1} e^{\mu z}\right.}\right] \\
& A_{34}(z)=\left[\frac{S_{-} E e^{-\mu z}-S_{+} E^{-1} e^{\mu z}}{2 c^{*}\left(E e^{-\mu z}-E^{-1} e^{\mu z}\right.}\right]
\end{aligned}
$$

where we define the following quantities

$$
\begin{aligned}
\Delta & \equiv d_{2}-d_{1} \\
Q & \equiv\left(\Delta^{2}+4|c|^{2}\right)^{1 / 2} \\
S_{ \pm} & \equiv \Delta \pm Q \\
\mu & =\gamma Q /\left(2 I_{0}\right)
\end{aligned}
$$

and $D$ and $E$ are constants of integration.

At this point, the problem has been transformed from a set of nonlinear differential equations (2.9) to another set of equations [(3.1) and (3.4)] which may be solved by fitting boundary conditions appropriate to the particular device under consideration.

We will first describe the application of this theory to the derivation of the reflectivity of a phase conjugate mirror with externally provided pumps. In this case, the amplitudes of all beams at their respective entrance faces are known.

We observe first of all that the power flux $\Delta=I_{2}(l)-I_{1}(0)$ $I_{4}(0)$ is known so we need only solve for $D, E$, and $c$ to finally obtain the phase conjugate reflectivity. The starting equations are the values of (3.4) at the boundaries $z=0$ and $z=l$. The conservation law (3.1a) is also used to express the unknown field quantities $A_{1}(l)$ and $A_{2}^{*}(0)$ in terms of $c, A_{3}(0)$, and known fields

$$
\begin{aligned}
& A_{12}(l)=-\left[\frac{S_{-} D e^{-\mu l}-S_{+} D^{-1} e^{\mu l}}{2 c^{*}\left(D e^{-\mu l}-D^{-1} e^{\mu l}\right)}\right]=\frac{c}{I_{2}(l)} \\
& A_{12}(0)=-\left[\frac{S_{-} D-S_{+} D^{-1}}{2 c^{*}\left(D-D^{-1}\right)}\right]=\frac{I_{1}(0)}{c^{*}-\rho^{*} I_{4}(0)} \\
& A_{34}(l)=\left[\frac{S_{-} E e^{-\mu l}-S_{+} E^{-1} e^{\mu l}}{2 c^{*}\left(E e^{-\mu l}-E^{-1} e^{\mu l}\right)}\right]=0 \\
& A_{34}(0)=\left[\frac{S_{-} E-S_{+} E^{-1}}{2 c^{*}\left(E-E^{-1}\right)}\right]=\rho .
\end{aligned}
$$

The procedure used to solve the equations is

1) Solve for $E$ in terms of $|c|^{2}$ using (3.9c).

$$
E=\left(\frac{S_{+}}{S_{-}}\right)^{1 / 2} e^{\mu l}=\left(\frac{\Delta+\left(\Delta^{2}+4|c|^{2}\right)^{1 / 2}}{\Delta-\left(\Delta^{2}+4|c|^{2}\right)^{1 / 2}}\right)^{1 / 2} e^{\mu l}
$$

2) Solve for $\rho$ in terms of $|c|^{2}$ using (3.9d) and (3.10).

$$
\rho=\frac{-2 c T}{\Delta T+\left(\Delta^{2}+4|c|^{2}\right)^{1 / 2}}
$$

where $T=\tanh \mu l$.

3) Solve for $D$ in terms of $|c|^{2}$ using (3.9a).

$$
D=\left(\frac{\Delta+\left(\Delta^{2}+4|c|^{2}\right)^{1 / 2}+2|c|^{2} / I_{2}(l)}{\Delta-\left(\Delta^{2}+4|c|^{2}\right)^{1 / 2}+2|c|^{2} / I_{2}(l)}\right)^{1 / 2} e^{\mu l} .
$$

4) Solve for $|c|^{2}$ using (3.9b), (3.11), and (3.12). We find that $|c|^{2}$ is given by the roots of the following equation:

$$
\begin{aligned}
\left(|c|^{2}\right. & \left.-I_{1}(0) I_{2}(l)\right)\left|\Delta T+\left(\Delta^{2}+4|c|^{2}\right)^{1 / 2}\right|^{2} \\
& +4|c|^{2}|T|^{2} I_{4}(0) I_{2}(l) \\
& +2|c|^{2} I_{4}(0)\left(\Delta^{2}+4|c|^{2}\right)^{1 / 2}\left(T+T^{*}\right)=0 .
\end{aligned}
$$

5) The phase conjugate reflectivity is then given by the squared modulus of (3.11).

When considering reflectivity as a function of the various input beam intensities, it is convenient to define the probe ratio $q$ :

$$
q \equiv \frac{I_{4}(0)}{I_{1}(0)+I_{2}(l)}
$$


so that only two parameters are required to describe the input beams: the probe ratio $q$ and the pump ratio $r=I_{2}(l) / I_{1}(0)$. In terms of these parameters we have

$$
\begin{aligned}
& I_{1}(0)=\frac{I_{0}}{(r+1)(q+1)} \\
& I_{2}(l)=\frac{r I_{0}}{(r+1)(q+1)} \\
& I_{4}(0)=\frac{q I_{0}}{(q+1)} .
\end{aligned}
$$

In Fig. 5, for example, we plot the reflectivity of a phase conjugate mirror as a function of the coupling strength $|\gamma l|$ for the case where the phase shift between the grating and the interference fringes is $5^{\circ}$. The intensities of the two pumping beams are equal $(r=1)$ and the probe intensity is 20 percent of the total pumping intensity $(q=0.2)$. The top of the graph corresponds to the reflectivity that would result if all the power of beam 2 were transferred to beam 3 . This is the maximum reflectivity consistent with the conservation laws (3.1). The peaks in the curve correspond to the poles in the reflectivity of a phase conjugate mirror with no pump depletion $\left(R=|\tanh (\gamma l / 2)|^{2}\right.$ for no phase shift between the grating and interference fringes). We have set the phase shift slightly nonzero to demonstrate the resultant damping of the oscillatory behavior. The peaks bend toward the right, probably since pump depletion causes high reflectivities to demand higher coupling strengths than are required for the same behavior in the undepleted phase conjugate mirror. The bending of the peaks can even be sufficient for bistability, as can be seen in the first two peaks of Fig. 5 .

In Fig. 6, we show a contour plot of phase conjugate reflectivity for $\gamma l=-3$, as a function of both pump and probe ratios. The first point to notice is the region of multistability, a direct result of the nonuniqueness of the solution of (3.13) for a certain range of parameters. Secondly, we observe that the reflectivity can remain finite as the pump ratio tends to infinity. This possibility for phase conjugation in the absence of pumping beam 2 has important consequences for the passive phase conjugate mirrors described below.

The basic physical difference between the two solution. surfaces of Fig. 6 lies in the relative phases of the two terms $A_{1} A_{4}^{*}$ and $A_{2}^{*} A_{3}$ in the interference factor $\left(A_{1} A_{4}^{*}+A_{2}^{*} A_{3}\right)$ which appears in the coupled wave equations (2.9). When the phase conjugate mirror is operating on the main surface, the one which extends over the entire $q-r$ plane, the phase conjugate beam is generated so that the interference pattern formed between itself and beam 2 is in phase with the interference pattern formed between the forward going beams 1 and 4 . On the secondary surface, these two terms are $\pi$ out of phase with each other, so that both the grating strength and the reflectivity are diminished.

It is often convenient to be able to examine the intensities of the various beams as a function of location $z$ in the crystal. For example, in the next section of this paper, we will consider several devices whose boundary conditions are given by the ratios of intensities of pumping beams. These ratios appear in functions whose zeros must be found to reach a solution.

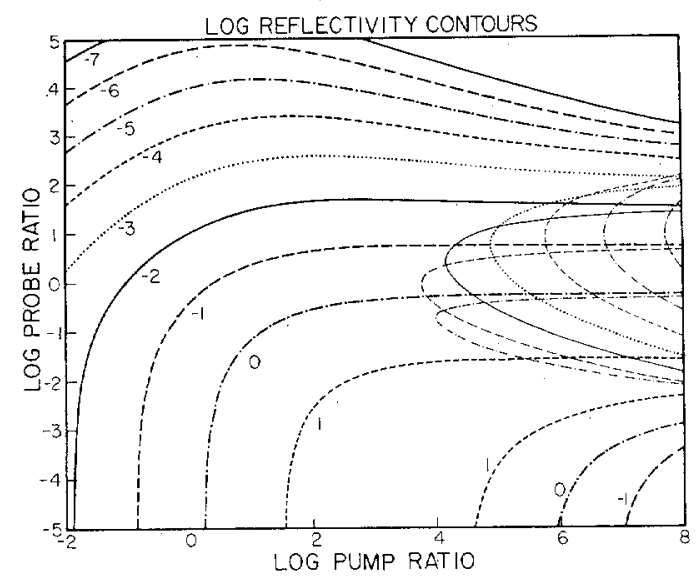

Fig. 6. Contour plot of phase conjugate reflectivity for $\gamma l=-3$ as a function of the pump ratio $\left(I_{2}(l) / I_{1}(0)\right)$ and the probe ratio $\left(I_{4}(l) /\right.$ $\left.\left[I_{1}(0)+I_{2}(l)\right]\right)$. The transmission grating is operative.

Occasionally, since the ratio of two negative numbers is positive, these functions will indicate solutions with negative intensities which nevertheless satisfy the boundary conditions. It is important in checking for these spurious solutions to have expressions for beam intensities as a function of $z$. These may be derived by using the amplitude ratio functions $A_{12}$ and $A_{34}$ in the conservation laws (3.1).

$$
\begin{aligned}
& I_{1}(z)=I_{12}(z) \frac{d_{2}-I_{34}(z) d_{1}}{1-I_{12}(z) I_{34}(z)} \\
& I_{2}(z)=\frac{d_{2}-I_{34}(z) d_{1}}{1-I_{12}(z) I_{34}(z)} \\
& I_{3}(z)=I_{34}(z) \frac{d_{1}-I_{12}(z) d_{2}}{1-I_{12}(z) I_{34}(z)} \\
& I_{4}(z)=\frac{d_{1}-I_{12}(z) d_{2}}{1-I_{12}(z) I_{34}(z)}
\end{aligned}
$$

where $I_{i j}=I_{i} / I_{j}$.

\section{The Reflection Grating}

Our procedure for the solution of the coupled wave equation (2.18) for the reflection grating differs significantly from that for the transmission grating, mainly because the spatially averaged intensity $I_{0}$ is no longer conserved. We have so far only been able to demonstrate complete solutions for $\pi / 2$ and zero phase shift $\varphi$ between index and interference patterns.

The first step is to observe that there is a simple solution for the interference term $g \equiv\left(A_{1} A_{4}^{*}+A_{2}^{*} A_{3}\right)$. Using (2.18) for negligible absorption we can immediately write

$$
\frac{d g}{d z}=\gamma g
$$

so that

$$
g=g_{0} e^{\gamma z} .
$$

This result can be used to simplify the coupled wave equations (2.18) to

$$
\frac{d A_{1}}{d z}=\frac{\gamma g_{0} e^{\gamma z}}{I_{0}} A_{4}
$$




$$
\begin{aligned}
& \frac{d A_{2}^{*}}{d z}=\frac{\gamma g_{0} e^{\gamma z}}{I_{0}} A_{3}^{*} \\
& \frac{d A_{3}}{d z}=\frac{\gamma g_{0} e^{\gamma z}}{I_{0}} A_{2} \\
& \frac{d A_{4}^{*}}{d z}=\frac{\gamma g_{0} e^{\gamma z}}{I_{0}} A_{1}^{*} .
\end{aligned}
$$

From these equations, we find that $I_{0}$ is given by

$$
I_{0}=\left(4\left|g_{0}\right|^{2} e^{\left(\gamma+\gamma^{*}\right) z}+K\right)^{1 / 2}
$$

where $K$ is a constant of integration. (3.19) can then be completely decoupled by combining (3.19b) with $(3.19 \mathrm{c})$ and (3.19a) with (3.19d) to give

$$
\begin{gathered}
\frac{d^{2} A_{1}}{d z^{2}}-\left[\gamma-\frac{2\left|g_{0}\right|^{2}\left(\gamma+\gamma^{*}\right) e^{\left(\gamma+\gamma^{*}\right) z}}{4\left|g_{0}\right|^{2}\left(\gamma+\gamma^{*}\right) e^{\left(\gamma+\gamma^{*}\right) z}+K}\right] \cdot \frac{d A_{1}}{d z} \\
-\frac{\left|g_{0} \gamma\right|^{2} e^{\left(\gamma+\gamma^{*}\right) z}}{4\left|g_{0}\right|^{2}\left(\gamma+\gamma^{*}\right) e^{\left(\gamma+\gamma^{*}\right) z}+K} A_{1}=0 \\
\frac{d^{2} A_{2}^{*}}{d z^{2}}-\left[\gamma-\frac{2\left|g_{0}\right|^{2}\left(\gamma+\gamma^{*}\right) e^{\left(\gamma+\gamma^{*}\right) z}}{4\left|g_{0}\right|^{2}\left(\gamma+\gamma^{*}\right) e^{\left(\gamma+\gamma^{*}\right) z}+K}\right] \cdot \frac{d A_{2}^{*}}{d z} \\
-\frac{\left|g_{0} \gamma\right|^{2} e^{\left(\gamma+\gamma^{*}\right) z}}{4\left|g_{0}\right|^{2}\left(\gamma+\gamma^{*}\right) e^{\left(\gamma+\gamma^{*}\right) z}+K} A_{2}^{*}=0
\end{gathered}
$$

$$
\frac{\gamma e^{\gamma z}}{I_{0}} d z=d u
$$

which gives

$$
u=\frac{1}{2\left|g_{0}\right|} \ln \left[\frac{2\left|g_{0}\right| e^{\gamma z}+\left(4\left|g_{0}\right|^{2} e^{\left(\gamma+\gamma^{*}\right) z}+K\right)^{1 / 2}}{2\left|g_{0}\right|+\left(4\left|g_{0}\right|^{2}+K\right)^{1 / 2}}\right] \text {. }
$$

Then (3.19) becomes

$$
\begin{aligned}
& \frac{d A_{1}}{d u}=g_{0} A_{4} \\
& \frac{d \dot{A}_{2}^{*}}{d u}=g_{0} A_{3}^{*} \\
& \frac{d A_{3}}{d u}=g_{0} A_{2} \\
& \frac{d A_{4}^{*}}{d u}=g_{0} A_{1}^{*} .
\end{aligned}
$$

Only if the phase shift $\varphi=\pi / 2$ so that the coupling constant $\gamma$ is real will we be able to work easily with the complex conjugates of the equations belonging to (3.24) to proceed by standard methods to the solution with $A_{3}(0)=0, A_{1}(0), A_{2}(l)$ and $A_{4}(l)$ known:

$$
\begin{aligned}
& A_{1}(u)=\frac{g_{0}^{1 / 2}\left[\left(\frac{A_{4}(l)}{g_{0}^{* 1 / 2}}+\frac{A_{1}(0)}{g_{0}^{1 / 2}} e^{-\left|g_{0}\right| u(l)}\right) e^{\left|g_{0}\right| u}+\left(\frac{A_{1}(0)}{g_{0}^{1 / 2}} e^{\left|g_{0}\right| u(l)}-\frac{A_{4}(l)}{g_{0}^{* 1 / 2}}\right) e^{-\left|g_{0}\right| u}\right]}{e^{\left|g_{0}\right| u(l)}+e^{-\left|g_{0}\right| u(l)}} \\
& A_{2}(u)=A_{2}(l) \frac{\left[e^{\left|g_{0}\right| u}+e^{-\left|g_{0}\right| u}\right]}{e^{\left|g_{0}\right| u(l)}+e^{-\left|g_{0}\right| u(l)}} \\
& A_{3}(u)=\left(\frac{g_{0}}{g_{0}^{*}}\right)^{1 / 2} A_{2}(l) \frac{\left[e^{\left|g_{0}\right| u}-e^{-\left|g_{0}\right| u}\right]}{e^{\left|g_{0}\right| u(l)}+e^{-\left|g_{0}\right| u(l)}} \\
& A_{4}(u)=\frac{g_{0}^{* 1 / 2}\left[\left(\frac{A_{4}(l)}{g_{0}^{* 1 / 2}}+\frac{A_{1}(0)}{g_{0}^{1 / 2}} e^{-\left|g_{0}\right| u(l)}\right) e^{\left|g_{0}\right| u}-\left(\frac{A_{1}(0)}{g_{0}^{1 / 2}} e^{\left|g_{0}\right| u(l)}-\frac{A_{4}(l)}{g_{0}^{* 1 / 2}}\right) e^{-\left|g_{0}\right| u}\right]}{e^{\left|g_{0}\right| u(l)}+e^{-\left|g_{0}\right| u(l)}} .
\end{aligned}
$$

$$
\begin{gathered}
\frac{d^{2} A_{3}}{d z^{2}}-\left[\gamma-\frac{2\left|g_{0}\right|^{2}\left(\gamma+\gamma^{*}\right) e^{\left(\gamma+\gamma^{*}\right) z}}{4\left|g_{0}\right|^{2}\left(\gamma+\gamma^{*}\right) e^{\left(\gamma+\gamma^{*}\right) z}+K}\right] \cdot \frac{d A_{3}}{d z} \\
-\frac{\left|g_{0} \gamma\right|^{2} e^{\left(\gamma+\gamma^{*}\right) z}}{4\left|g_{0}\right|^{2}\left(\gamma+\gamma^{*}\right) e^{\left(\gamma+\gamma^{*}\right) z}+K} A_{3}=0 . \\
\frac{d^{2} A_{4}^{*}}{d z^{2}}-\left[\gamma-\frac{2\left|g_{0}\right|^{2}\left(\gamma+\gamma^{*}\right) e^{\left(\gamma+\gamma^{*}\right) z}}{4\left|g_{0}\right|^{2}\left(\gamma+\gamma^{*}\right) e^{\left(\gamma+\gamma^{*}\right) z}+K}\right] \cdot \frac{d A_{4}^{*}}{d z} \\
-\frac{\left|g_{0} \gamma\right|^{2} e^{\left(\gamma+\gamma^{*}\right) z}}{4\left|g_{0}\right|^{2}\left(\gamma+\gamma^{*}\right) e^{\left(\gamma+\gamma^{*}\right) z}+K} A_{4}^{*}=0 .
\end{gathered}
$$

For $\gamma+\gamma^{*}=0$, corresponding to zero spatial phase shift, these equations have constant coefficients and can be solved by elementary techniques. Otherwise, there is no clear way to integrate them. Fortunately, for the phase shift of greatest interest to us in the PR effect, $\varphi=\pi / 2$, there is an alternative way to proceed to a solution. We make a change of variable from $z$ to $u$ defined by
The phase conjugate reflectivity is thus given by

$$
R=\frac{I_{2}(l)}{I_{4}(l)} \tanh ^{2}\left(\left|g_{0}\right| u(l)\right) .
$$

To evaluate $\left|g_{0}\right| u(l)$ we substitute (3.25) into (3.18), using the definition of $g$, and evaluate at $z=0$ and $l$ to obtain two equations in $g_{0},\left|g_{0}\right|$, and $\left|g_{0}\right| u(l)$. Solving for $g_{0}$, taking the magnitude, and then eliminating $\left|g_{0}\right|$ yields

$$
\begin{aligned}
\sinh \left|g_{0}\right| u(l) & =\frac{\sqrt{I_{1}(0) I_{4}(l)}\left(1 \pm e^{-\gamma l}\right)}{I_{1}(0)+\left(I_{2}(l)+I_{4}(l)\right) e^{-\gamma l}} \\
& =\frac{\sqrt{q(1+r)}\left(1 \pm e^{-\gamma l}\right)}{1+[r+q(1+r)] e^{-\gamma l}}
\end{aligned}
$$

where now $q=I_{4}(l) /\left[I_{1}(0)+I_{2}(l)\right]$. Of the two solutions, only one (the minus sign) reduces to the undepleted pumps solution (2.15) as $q \rightarrow 0$. Fig. 7 is a contour plot of this solution for the phase conjugate reflectivity as a function of pump and probe ratios, for $\gamma l=-3$. In contrast to the case of the 


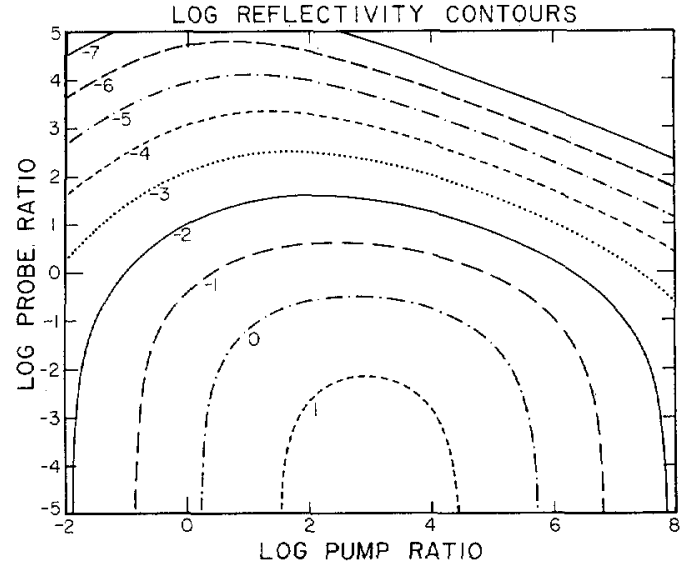

Fig. 7. Contour plot of phase conjugate reflectivity for $\gamma l=-3$ as a function of the pump ratio $\left(I_{2}(l) / I_{1}(0)\right)$ and the probe ratio $\left(I_{4}(l) /\right.$ $\left.\left[I_{1}(0)+I_{2}(l)\right]\right)$. The reflection grating is operative.

transmission grating (Fig. 6), the reflectivity due to the reflection grating goes to zero in the limit of the infinite pump ratio.

\section{Passive Phase Conjugate Mirrors and Lasers with Dynamic Intracavity Distortion CORRECTION CAPABILITY}

Certain PR materials such as $\mathrm{BaTiO}_{3}$ and $\mathrm{SBN}$ are endowed with electrooptic coefficients so large $\left(\approx 1000 \mathrm{pV} \cdot \mathrm{m}^{-1}\right)$ that values of $\gamma$ of the order of $10^{3} \mathrm{~m}^{-1}$ are easily obtainable with milliwatt input beams. Not only does this make it possible to build phase conjugate mirrors with large reflectivities but also, it enables the construction of phase conjugate resonators [59][65] (PCR's), as well as other useful resonator devices such as passive phase conjugate mirrors (PPCM's) and unidirectional ring resonators. The main advantage of the PPCM is that it obviates the high quality externally provided pumping beams needed for conventional four-wave mixing phase conjugate mirrors. In this section we describe the theory and recent experimental demonstrations of several PPCM's. Novel interpretations of photorefractive crystals as error correcting optical elements and as double phase conjugate mirrors will also be introduced.

\section{The Linear and Semilinear Passive Phase Conjugate Mirrors}

The first passive PR phase conjugate mirror was the linear mirror [42]-[44], a device consisting of an appropriately oriented PR crystal lying in a cavity bounded by two ordinary mirrors [Fig. 8(a)]. It has been used in imaging experiments and as the end mirror of an argon ion laser. It has also proved to be amenable to analysis by the nonlinear PR four-wave mixing theory described in the preceding paragraphs. The theory of the device has been useful in developing an understanding of its more unusual features. For example, we can understand the fact that under certain circumstances, it is possible to maintain operation when one of the external cavity mirrors is removed.

The physical basis for buildup of oscillation in the linear

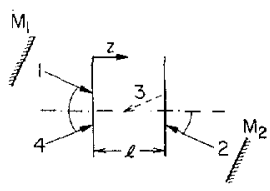

(a)

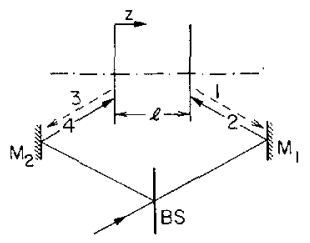

(b)

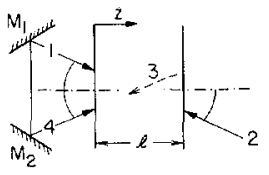

(c)

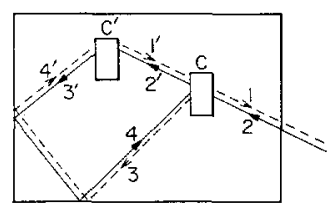

(d)

Fig. 8. (a) Geometry of the linear passive phase conjugate mirror. The probe is beam 4 and its phase conjugate is beam 3 . The two pumping beams, 1 and 2, oscillate in the cavity bounded by mirrors $M_{1}$ and $M_{2}$. (b) Geometry of a four-wave mixing oscillator which can produce output beams which are not phase conjugates of the input beams. (See text.) Beams 2 and 4 are provided from an external source and beams 1 and 3 build up via four-wave mixing. (c) Geometry of the ring passive phase conjugate mirror. Consistent with the convention that the intensity of beam 3 should be zero at its entrance face, the probe is designated as beam 2. Pump beam 4 is provided by reflection of the probe beam by mirrors $M_{1}$ and $M_{2}$. The second pump beam 3 , and the phase conjugate beam 1 are self-induced by the nonlinear medium. (d) Geometry of the two-interaction-region mirror. It consists of a ring mirror (using interaction region $C$ ) with a double phase conjugate mirror (using interaction region $C^{\prime}$ ) in its feedback loop. Both interaction regions are inside a single crystal whose boundary (large rectangle) gives rise to feedback by total internal reflection.

PPCM is the phenomenon of light amplification by two-bean. coupling. This effect occurs when the phase shift between the index grating and the interference pattern is nonzero. The $c$-axis of the crystal is oriented so that light in beam 1 is amplified by two-beam coupling from input beam 4 and is fed back by successive reflections from mirrors $M_{2}$ and $M_{1}$. Oscillation continues to build up until steady state is reached for beams 1 and 2 which are now pumping the crystal as a phase conjugate mirror for input beam 4 . We show here how to derive the reflectivity of the device (i.e., the intensity of beam 3 divided by the intensity of beam 4) in the slowly varying field, negligible linear absorption and single grating approximations. Because the pumping beams are derived from and fed by the signal beam itself, the commonly used undepleted pumps approximation is inappropriate. Thus, we use the analysis of Section III where we derived the reflectivity of a PR phase conjugate mirror without assuming undepleted 
pumps, but assuming knowledge of their input intensities. From now on except where otherwise noted, the theory for the transmission grating is used and all intensities are normalized by the conserved quantity $I_{0}$. When discussing the reflection grating, this normalization is not used.

Because the boundary conditions in the linear PPCM are not the input intensities $I_{1}(0)$ and $I_{2}(l)$, but rather the reflectivities of mirrors $M_{1}$ and $M_{2}$, some further development of the theory is required. We make use of the functions $A_{12}$ and $A_{34}$ to fit these new boundary conditions [see (3.4), (3.10), (3.12)] .

$$
M_{1}=I_{12}(0)=\frac{|c|^{2}}{I_{2}(l)^{2}}\left|\frac{T+\left(\Delta^{2}+4|c|^{2}\right)^{1 / 2}}{\Delta T+\left(\Delta^{2}+4|c|^{2}\right)^{1 / 2}+2|c|^{2} T / I_{2}(l)}\right|^{2}
$$

$$
M_{2}=1 / I_{12}(l)=I_{2}(l)^{2} /|c|^{2} .
$$

Since

$$
I_{2}(l)=(\Delta+1) / 2
$$

and

$$
|c|^{2}=(\Delta+1)^{2} /\left(4 M_{2}\right)
$$

we see that (4.1) is really a function of the single variable $\Delta$, together with the known quantities $\gamma l, M_{1}$, and $M_{2}$. Similarly, the reflectivity $R$ may be rewritten as a function of these same variables. We have

$$
\begin{aligned}
M_{1} M_{2} & =I_{12}(0) / I_{12}(l) \\
& =\left|\frac{T+\left[\Delta^{2}+(\Delta+1)^{2} / M_{2}\right]^{1 / 2}}{\Delta T+\left[\Delta^{2}+(\Delta+1)^{2} / M_{2}\right]^{1 / 2}+(1+\Delta) T / M_{2}}\right|^{2}
\end{aligned}
$$

where

$$
\begin{aligned}
& T=\tanh \left(\frac{\gamma l}{2}\left[\Delta^{2}+(\Delta+1)^{2} / M_{2}\right]^{1 / 2}\right) \\
& R=I_{34}(0)=\frac{(1+\Delta)^{2}|T|^{2}}{M_{2}\left|\Delta T+\left[\Delta^{2}+(\Delta+1)^{2} / M_{2}\right]^{1 / 2}\right|^{2}} .
\end{aligned}
$$

Thus, the reflectivity of the PPCM may be found by solving (4.3) for $\Delta$ and then using the resultant value(s) in (4.5) for $R$. We note that (4.3) may have multiple roots.

We show in Fig. 9 a contour plot of the reflectivity $R$ as a function of $M_{1}$ and $M_{2}$ for a particular value of the coupling strength $\gamma l=-3$ (i.e., with the $\pi / 2$ phase shift typical of photorefractive materials). We see that towards the left of this plot the reflectivity can be multivalued, and also that when $M_{2}$ is high the reflectivity remains high even when $M_{1}$ is small. We shall show that there is a threshold coupling strength above which it is possible to obtain finite reflectivities even in the absence of mirror $M_{1}$.

But first, we consider the threshold coupling strength for the buildup of oscillation from zero oscillation intensity in the $M_{1}-M_{2}$ cavity. This corresponds to taking $I_{1}(0)=I_{2}(l)=0$, that is, $\Delta=-1$. From (4.3) the threshold may be obtained as

$$
M_{1} M_{2}=\exp \left[\left(\gamma+\gamma^{*}\right) l\right] .
$$

This fits in well with the heuristic expansion of oscillation buildup given earlier; the gain in the crystal simply has to be sufficient to overcome the losses due to the mirrors $M_{1}$ and $M_{2}$. Since the threshold depends only on the real part of the coupling strength, it follows that a nonlinear medium with no phase shift between the index grating and the interference pattern will not support operation beginning from zero oscillation strength. This is because of the absence in these materials of unidirectional two-beam coupling.

In addition, we see that no buildup of operation from zero oscillation strength is possible in the absence of mirror $M_{1}$ even when $\gamma l$ does have a real part. However, by providing a seed beam in the $M_{2}$-crystal cavity, so that the initial probe ratio is not infinite, it is possible in some cases to build up oscillation in the absence of mirror $M_{1}$. We call this device the semilinear mirror. It will not start by itself, but once initiated, it keeps going. Such behavior could have been anticipated from Fig. 6 which indicated that finite reflectivities were available at infinite pump ratio. This is exactly the situation confronting us here. It can also be shown that semilinear operation is not possible with the use of four-wave mixing via the reflection grating. A semilinear mirror using the reflection grating would have the interference term $g\left[A_{1} A_{4}^{*}+A_{2}^{*} A_{3}\right]$ equal to zero either at the plane $z=l$ or at the plane $z=0$ because we must have either $A_{4}(l)$ and $A_{2}(l)$ or $A_{1}(0)$ and $A_{3}(0)$ equal to zero. Since $g=g_{0} e^{\gamma z}$ (3.18) if $g$ is zero at one plane, it must be zero everywhere, so that no coupling is possible.

The theory of the transmission grating with $M_{1}=0$ implies [see (4.3)] that

$$
\begin{gathered}
\tanh \left(-\frac{\gamma l}{2}\left[\Delta^{2}+(\Delta+1)^{2} / M_{2}\right]^{1 / 2}\right) \\
=\left[\Delta^{2}+(\Delta+1)^{2} / M_{2}\right]^{1 / 2}
\end{gathered}
$$

so that $\Delta$ may found from the solution of the quadratic equation

$$
\Delta^{2}+\frac{(\Delta+1)^{2}}{M_{2}}=a^{2}
$$

where $a$ is simply related to the coupling constant $\gamma l$ by

$$
\tanh \left(-\frac{\gamma l}{2} a\right)=a
$$

The reflectivity can therefore be written in closed form as

$$
R=\left(\frac{M_{2}^{1 / 2} \pm\left[a^{2}\left(1+M_{2}\right)-1\right]^{1 / 2}}{M_{2}+2 \mp M_{2}^{1 / 2}\left[a^{2}\left(1+M_{2}\right)-1\right]^{1 / 2}}\right)^{2}
$$

so that the device is at threshold with reflectivity $R=R_{t}$

$$
R_{t}=\frac{M_{2}}{\left(M_{2}+2\right)^{2}}
$$

when $a^{2}$ equals $a_{t}^{2}$ 


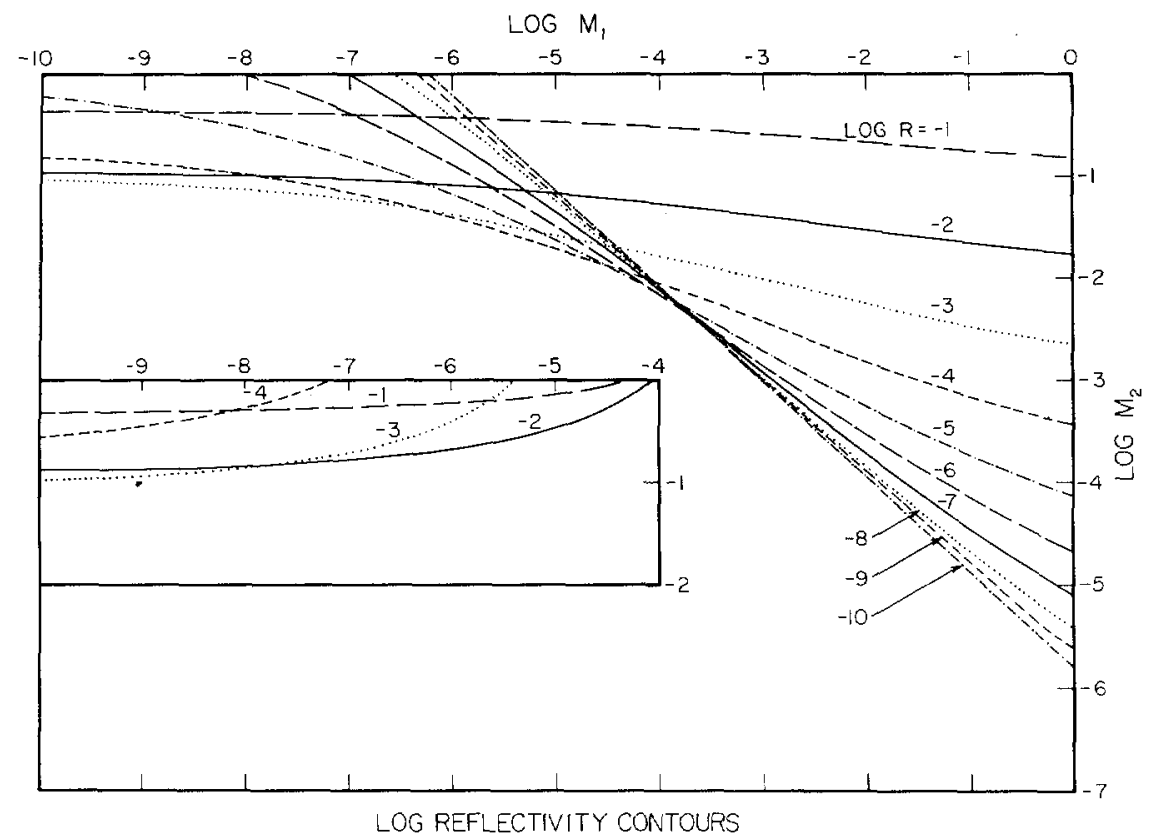

Fig. 9. Contour plots of the reflectivity of the passive phase conjugate mirror. Equality of contour levels in the region where the function is multivalued is indicated by equality of line form (dashes, dots, etc.). The coupling strength $\gamma l=-3$. For the sake of clarity some of the contours at low $M_{1}$ and high $M_{2}$ have been redrawn in an inset.

$$
a_{t}^{2}=\frac{1}{\left(1+M_{2}\right)} .
$$

In terms of $\gamma$ this threshold is given by

$$
\gamma l_{t}=\left(1+M_{2}\right)^{1 / 2} \ln \left[\frac{\left(1+M_{2}\right)^{1 / 2}-1}{\left(1+M_{2}\right)^{1 / 2}+1}\right] .
$$

It is possible to show that of the two possible values of abovethreshold reflectivity (4.10) only the one associated with the upper sign is stable. This mode of operation, without $M_{1}$, has been observed in our laboratory and, as we describe below, has been used in the PPCM as an end mirror for an argon ion laser.

Having examined the theory of this device, we now turn to results of two experimental verifications of the phase conjugating nature of the linear PPCM. The first is a demonstration of the phase conjugate imaging property of the PPCM. The second is an experiment showing intracavity distortion correction in an argon ion laser operated with one of its end mirrors replaced by linear and semilinear PPCM's.

In the first experiment [44] (Fig. 10) the expanded and spatially filtered output of a dye laser illuminated a transparency $T$ [Fig. 11(a)]. The beam passed through lens $L_{2}$ and converged on the linear mirror, consisting of a single poled crystal of barium titanate lying in the $M_{1}-M_{2}$ resonator cavity. The reflected beam was picked off by a beam splitter BS and photographed at the location where a phase conjugate image would be expected. The result is shown in Fig. 11(b). The phase conjugating behavior of the linear mirror is quite evident. Intensity patterns of the oscillation beams in the $M_{1}-M_{2}$ cavity are shown in Fig. 11(c) and (d). These were photographs of the intensities at mirrors $M_{1}$ and $M_{2}$, respectively. The information in the probe beam has been filtered out, leaving specklelike patterns due to optical inhomogeneities in the crystal.

The arrangement for the $\mathrm{CW}$ argon ion laser experiment [43] is shown in Fig. 12. Lasing was initially induced at the high

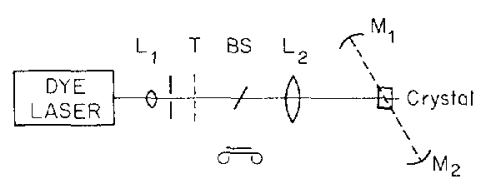

Fig. 10. The experimental arrangement used to demonstrate phase conjugation in the linear mirror. The dye laser used was a Spectra Physics 380 ring laser with rhodamine $6 G$ at $579.2 \mathrm{~nm}$ is single longitudinal mode. Using a Cartesian coordinate system with the absicissa coincident with the beam direction, the locations of the elements measured in centimeters were: $20 \times$ beam expander $L_{1}(-64,0)$, transparency $T(-55,0)$, beam splitter for observing phase conjugate reflection $\mathrm{BS}(-38,0), 14 \mathrm{~cm}$ focal length lens $L_{2}(-20,0)$, barium titanate crystal $(0,0), 50 \mathrm{~cm}$ radius concave mirror $M_{1},(-28,11), 50$ $\mathrm{cm}$ radius concave mirror $M_{2}(30,-13)$. The $c$-axis of the crystal pointed in the direction of the vector $(0.94,0.35)$. This crystal measured $7 \times 4.5 \times 4 \mathrm{~mm}$ and was poled into a single domain so that the $c$-axis was parallel to the $4 \mathrm{~mm}$ side.

gain line, $488.0 \mathrm{~nm}$, between mirror $M_{3}$ and beam splitter BS [Fig. 12(a)]. Light transmitted through the beam splitter caused oscillation in the PPCM, the resonator consisting of a $\mathrm{BaTiO}_{3}$ crystal and mirrors $M_{1}$ and $M_{2}$. Reflecting mirror $M_{4}$ was used to assist in the buildup of oscillation. With oscillation established between $M_{1}$ and $M_{2}$, the beam splitter and the retroreflecting mirror $M_{4}$ were removed, as shown in Fig. 12(b). The starting procedure described above was required since the coherence of the fluorescence was insufficient to allow the formation of the required refractive index grating in the crystal. Once the grating was established, the configuration of Fig. 12(b) corresponds to an equilibrium state, and the grating in the crystal was continuously maintained by the very beams which it coupled together.

The theory (4.13) indicates that there is a certain two-beam coupling strength in the crystal, above which it is possible to maintain oscillation between the crystal and $M_{2}$ even in the absence of mirror $M_{1}$. We were able to demonstrate such oscillation in our laser. Fig. 12(c) depicts the starting arrangement. Once oscillation involving mirror $M_{2}$ was established, 


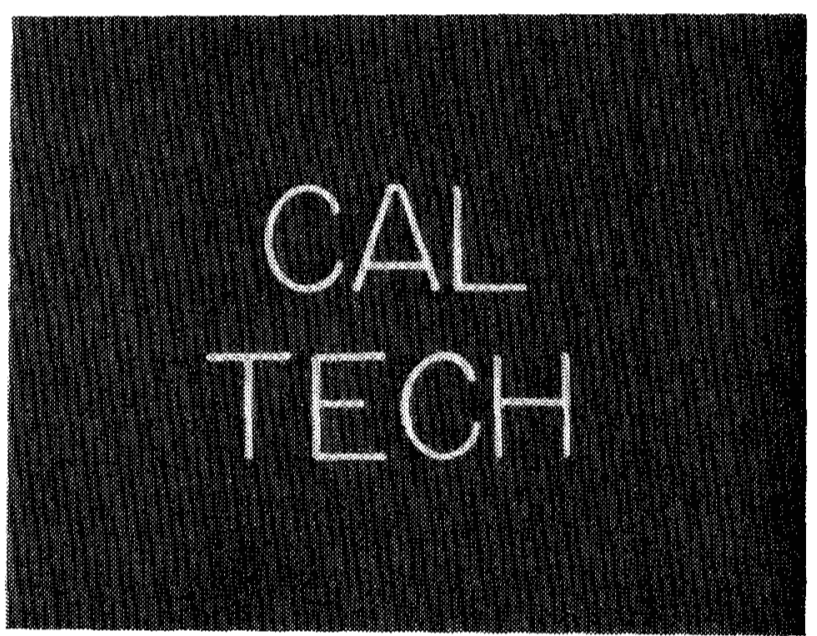

$\stackrel{\mathrm{mm}}{\overparen{\mathrm{m}}}$

(a)

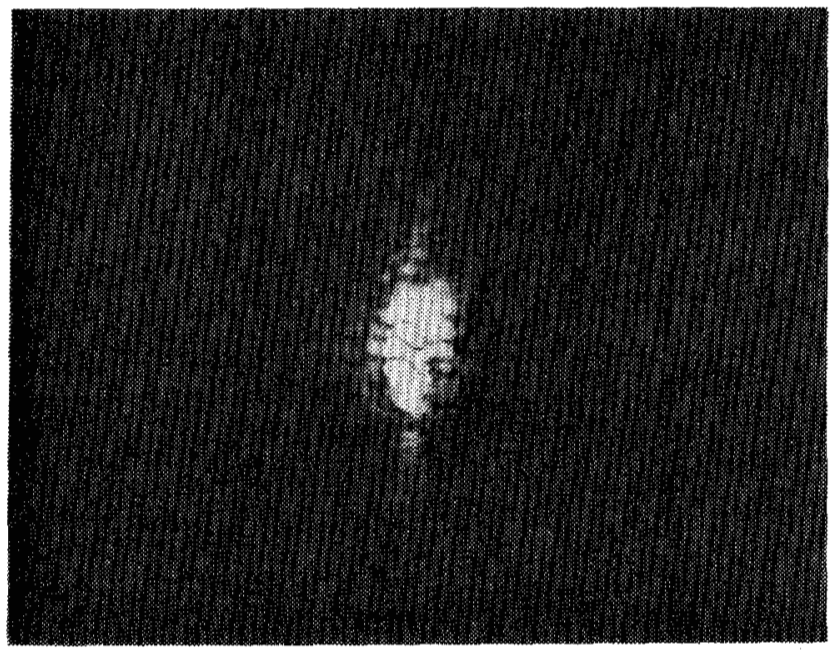

$\stackrel{\longmapsto}{2 \mathrm{~mm}}$

(c)

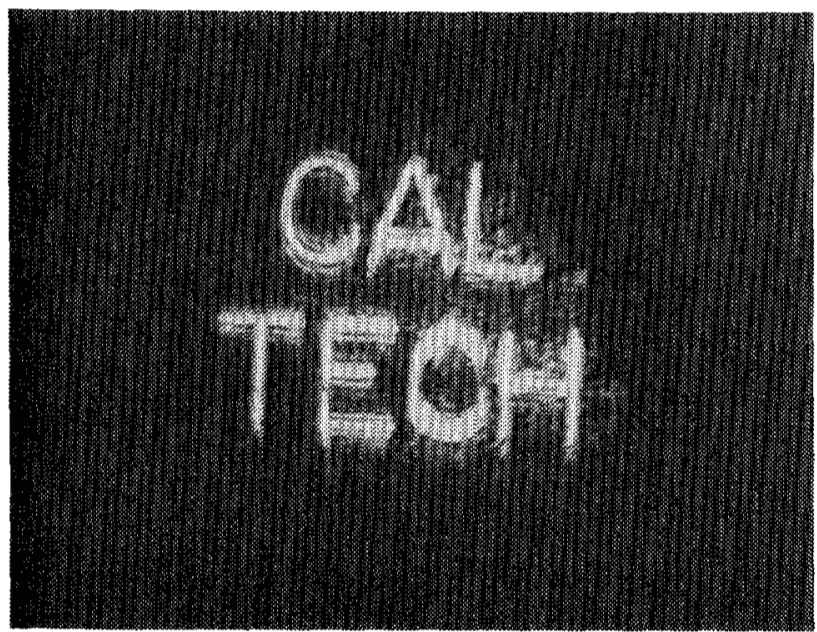

$\stackrel{-1 \mathrm{~mm}}{\longmapsto}$

(b)

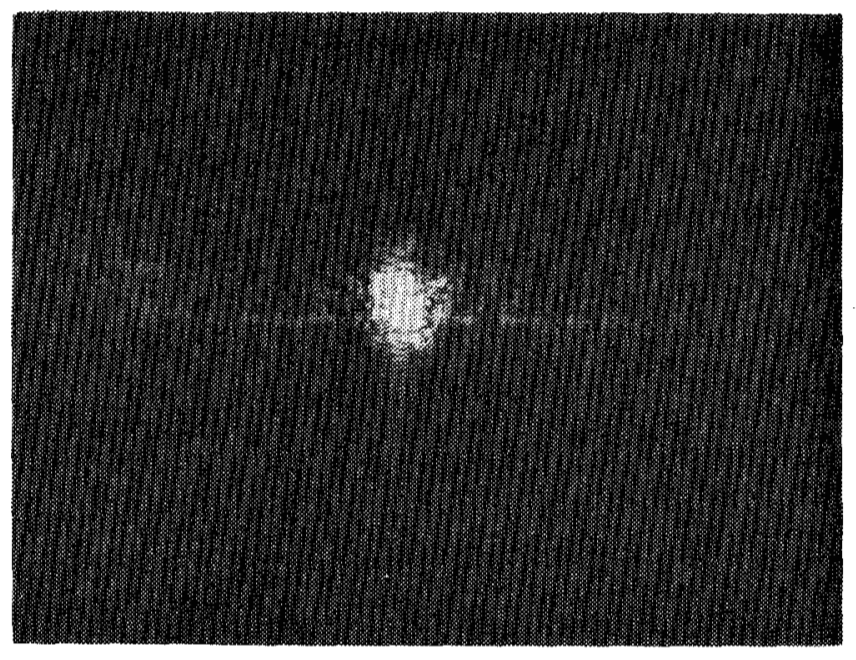

$\stackrel{\mathrm{mm}}{2}$

(d)

Fig. 11. Results of the linear mirror phase conjugation experiment. (a) The transparency $T$. (b) The phase conjugate beam picked off by beam splitter BS. (c) The intensity pattern of the oscillation beam on mirror $M_{1}$. (d) The intensity pattern of the oscillation beam on mirror $M_{2}$.

the beam splitter and mirror $M_{4}$ were removed and the laser continued to oscillate, as shown in Fig. 12(d).

To demonstrate the distortion correction capability of the laser with the PPCM, we operated it in the configuration of Fig. 12(d) with a severe distortion placed between the barium titanate crystal and the laser gain medium. Fig. 13(b) shows a photograph of the intensity pattern of the beam exiting through mirror $M_{3}$. Operating the laser in a conventional fashion with the crystal replaced by a high-reflectivity dielectric mirror and with the distortion in the beam path gave rise to the beam shown in Fig. 13(a). The compensation effect of the PPCM is evident. The power output at $38 \mathrm{~A}$ laser tube current in the conventional resonator with the distortion inside was about $1 \mathrm{~mW}$ compared to about $500 \mathrm{~mW}$ with the PPCM.

The loss of independence of the pump beams in the linear mirror results in one difference from a regular phase conjugate resonator. Longitudinal modes are present in the cavity, corresponding to the normal modes observed in a standing wave resonator and have been observed with an optical spectrum analyzer. The correspondence between the modes of an ordinary laser and a phase conjugate resonator laser is not yet well understood and is the subject of ongoing investigations.

\section{The Double Phase Conjugate Mirror or Intracavity Distortion Correction Device}

In the preceding discussion, the laser with dynamic holographic intracavity distortion correction capability was interpreted as consisting of an ordinary end mirror, an argon ion laser gain medium, and a semilinear PPCM. We offer here two alternative interpretations. In the first, the laser [Fig. 12(d)] is viewed as consisting of an ordinary end mirror $M_{1}$, an argon 
(a)

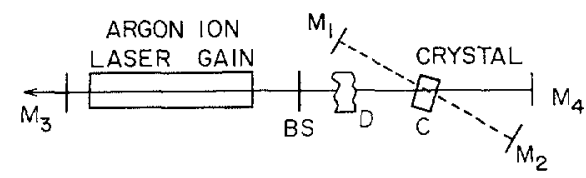

(b)

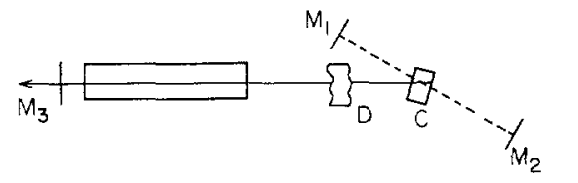

(c)

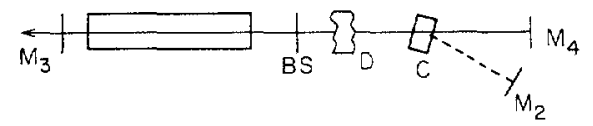

(d)

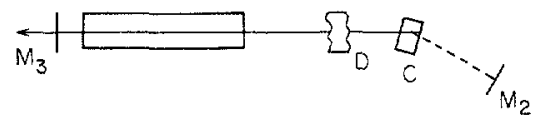

Fig. 12. Passive phase conjugate resonator laser. (a) Starting configuration. Mirror $M_{3}$ was the standard high radius of curvature output mirror of the Spectra Physics argon ion laser. The distance from it to the barium titanate crystal $C$ was $220 \mathrm{~cm}$. Mirror $M_{1}$ is flat and 50 percent reflecting, and mirror $M_{2}$ was concave $5 \mathrm{~cm}$ radius of curvature and highly reflecting. The distances between mirror $M_{2}$ and the crystal, and mirror $M_{1}$ and the crystal were both $4.5 \mathrm{~cm}$. None of these parameters was critical to the operation of the laser; for example, $M_{2}$ could be replaced at the same location by a $50 \mathrm{~cm}$ concave mirror. The position of the intracavity distortion $D$ is indicated on the figure. (b) Operating configuration. The crystal is pumped as a phase conjugate mirror by the beams shown dashed in the $M_{2}-M_{1}$ cavity. (c) Starting configuration for laser without mirror $M_{1}$. (d) Operating configuration for laser without $\operatorname{mirror} M_{1}$.

ion laser gain medium, the crystal as an intracavity distortion correction device and another ordinary mirror, $M_{3}$. The second interpretation comes from noting that the aberration compensation observed in the argon ion laser indicated that each of the oscillations-one in the $M_{1}$-crystal arm and the second in the $M_{3}$-crystal arm-was composed of two oppositely traveling waves which were phase conjugates of each other [(Fig. 12(d)]. The crystal thus acted simultaneously as a phase conjugate mirror to the two beams which were incident on it, coupling, in the process, the two arms to each other. We call a crystal operating in such a manner a double phase conjugate mirror.

Are the oscillation beams in a crystal with $I_{1}(0)=I_{3}(l)=0$ always phase conjugates of each other? One possibility is that oscillation beams contribute most to the four-wave mixing process and experience maximum gain when the spatial overlap of counterpropagating beams is maximum, that is, when they are phase conjugates of each other. However, this explanation is inconsistent with the behavior of another device [Fig. 8(b)] which we built [45] in the hope that it would be a phase conjugate mirror. A signal beam was incident on a beam splitter and the transmitted beam was directed by mirror $M_{1}$ onto a barium titanate crystal as beam 2 . The reflection from the beam splitter was directed by mirror $M_{2}$ onto the crystal as beam 4 . The theory developed below indicates that with sufficient coupling strength and $\pi / 2$ phase shift between the interference fringes and refractive index fringes, oscillation beams 1 and 3 can be expected to build up via gratings written between beams 1 and 4 and beams 2 and 3 . The wave vector of the beam 1-beam 4 grating must be the same as that of the beam 2-beam 3 grating so that both combine to form a single

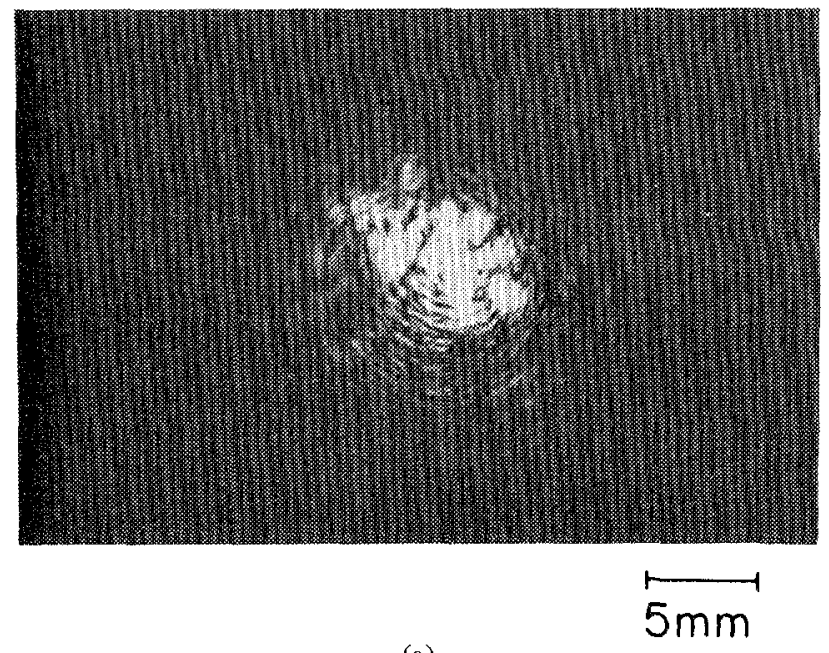

(a)

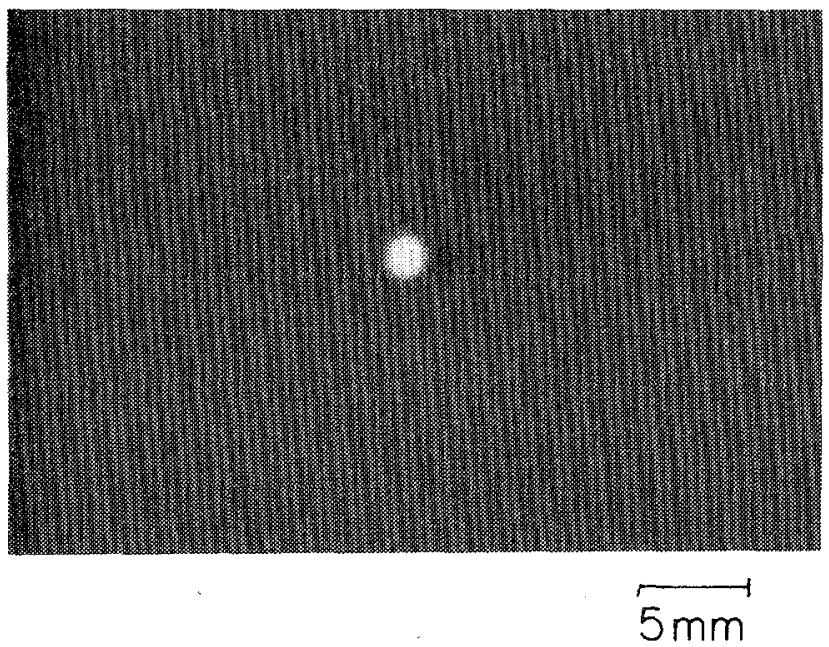

(b)

Fig. 13. (a) Photograph of the output of regular laser (i.e., crystal replaced by high reflectivity dielectric mirror) containing an intracavity distortion. The distance from the output mirror $M_{3}$ is $1 \mathrm{~m}$. The laser intensity is $1 \mathrm{~mW}$, obtained at $38 \mathrm{~A}$ laser tube current. (b) The output of the passive phase conjugate resonator laser containing the intracavity distortion. The distance from the output mirror $M_{3}$ is $1 \mathrm{~m}$. The laser intensity here is $3 \mathrm{~mW}$, obtained at only 21 A laser tube current. The power output at $38 \mathrm{~A}$ is $500 \mathrm{~mW}$.

grating coupling all four beams. Thus we require

$$
k_{1}-k_{4}=k_{3}-k_{2}
$$

where $k_{j}$ is the wave vector of beam $j$. In conventional applications of four-wave mixing $k_{1}, k_{2}$, and $k_{4}$ are fixed so that (4.15) gives a unique value for $k_{3}$, the wave vector of the phase conjugate beam. Since only $\boldsymbol{k}_{2}$ and $\boldsymbol{k}_{4}$ are fixed in the device of Fig. 8(b), however, there is an extra degree of freedom manifested in the expected and observed appearance of beams 1 and 3 as cones of light with axis $\boldsymbol{k}_{2}-\boldsymbol{k}_{4}$ and surfaces including both vectors $k_{2}$ and $\boldsymbol{k}_{4}$. This means that self-induced oscillation by four-wave mixing does not always require that the counterpropagating beams be phase conjugates of each other.

The full linear and semilinear mirrors involve feedback to the crystal of the oscillation beams while the beam splitter device described above does not: in the linear and semilinear mirrors, beam 1 is reflected into beam 2 and vice versa by 
external mirrors while in the beam splitter device, the oscillation beams are lost to the outside world. It is feedback that gives phase conjugate oscillations an advantage in gain over other kinds of oscillation. We will return to this point when we discuss the ring mirror in a later section.

When we come to design optical systems and circuits using a double phase conjugating PR crystal as a compensating element, it will be advantageous to have an expression for its transmissivity. From (4.2) and (4.6) we know that

$$
\begin{aligned}
4|c|^{2} & =a^{2}-\Delta^{2} \\
& =a^{2}\left[I^{2}(l)+I_{4}(0)\right]^{2}-\left[I_{2}(l)-I_{4}(0)\right]^{2} .
\end{aligned}
$$

The transmissivities in each direction actually turn out to be identical

$$
\begin{aligned}
\mathrm{T} & \equiv \frac{I_{1}(l)}{I_{4}(0)}=\frac{I_{3}(0)}{I_{2}(l)}=\frac{|c|^{2}}{I_{4}(0) I_{2}(l)} \\
& =\frac{a^{2}\left[q^{-1 / 2}+q^{1 / 2}\right]^{2}-\left[q^{-1 / 2}-q^{1 / 2}\right]^{2}}{4}
\end{aligned}
$$

where we have used the fact that the probe ratio $q$ is simplified in this case to $I_{4}(0) / I_{2}(l)$. The device may then also be interpreted as an aberration correcting absorber, whose absorption depends on the intensity ratio of the two entering beams. The lowest possible threshold for this device obtained from the definition of $a(4.9)$, is $\gamma l_{t}=-2$.

The simplest optical system involving the double phase conjugate mirror, apart from the folded cavity laser described above [Fig. 14(a)], is a ring laser with intracavity distortion correction capability [Fig. 14(b)]. The optical feedback provided in the ring cavity should encourage phase conjugate operation. If the single-pass gain is assumed to be $G$, so that $I_{4}(0)=G I_{1}(l)$ and $I_{2}(l)=G I_{3}(0)$, then after fitting these boundary conditions, we find that the equilibrium value of $q$ is given by

$$
q=2 \xi-1 \pm 2[\xi(\xi-1)]^{1 / 2}
$$

where

$$
\xi=\left(1-G^{-1}\right) /\left(1-a^{2}\right) .
$$

Such a laser is therefore bistable: (4.18) offers two solutions which are reciprocals of each other. This is can be understood on the basis of the symmetry of (4.17) in $q$ and $q^{-1}$. The gain threshold for this ring laser as derived from the requirement $\xi \geqslant 1$ is simply $G a^{2} \geqslant 1$ [see (4.18)].

\section{The Ring Passive Phase Conjugate Mirror}

In this section we describe another kind of PPCM. Unlike the linear PPCM's it generates only one of its pumping beams via nonlinear optical interactions. The results of a theoretical analysis of this device are shown as well as experimental verification of its action as a phase conjugate mirror [45].

In the basic implementation of this device [Fig. 8(c)] the signal beam 2 passes through a photorefractive medium and returns to it as pumping beam 4 around an optical ring cavity, here represented by mirrors $M_{1}$ and $M_{2}$. It may be advantageous to use curved mirrors or intracavity lenses to minimize diffractive loss of any spatial information on the signal beam.

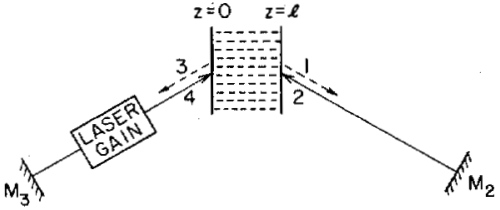

(a)

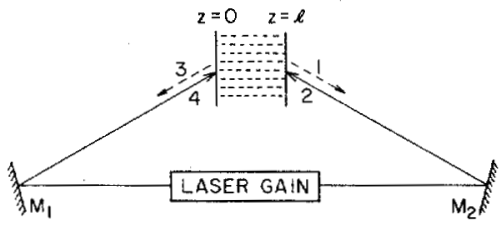

(b)

Fig. 14. PR crystal acting as a double phase conjugate mirror in (a) the semilinear mirror, (b) a ring laser with dynamic intracavity distortion correction capability.

The possibility then arises that the nonlinear optical coupling in the crystal may be such that both the second pumping beam 3 and the phase conjugate beam 1 build up as oscillation beams in the ring cavity. We now turn to a theoretical examination of the possibility. As in the case of the linear mirror, use of the undepleted pumps approximation is inappropriate and here, too, we use the depleted pumps analysis of Section III. After fitting boundary conditions of this device, named the ring mirror, we find that buildup of oscillation in the ring is in fact possible when the spatial phase shift between the holographic refractive index grating and the light interference pattern is nonzero so that advantage is taken of unidirectional beam coupling effects typical of real time holography in PR crystals.

In previous calculations, beam 3 has been the phase conjugate beam. Here, however, we designate beam 1 as the phase conjugate, because we wish to retain the convention that $I_{3}(l)=$ 0 , so that previous results may be directly adapted to the solution required here. The phase conjugate reflectivity is then going to be $R=I_{12}(l)$. Let the product of the intensity reflectivities of the feedback mirrors $M_{1}$ and $M_{2}$ be $M$ so that the appropriate boundary conditions for the ring mirror [Fig. $8(\mathrm{c})]$ are

$$
\begin{aligned}
& I_{4}(0) / I_{2}(0)=M \\
& I_{1}(0) / I_{3}(0)=M .
\end{aligned}
$$

Equation (3.16) supplies the required intensities in terms of $I_{12}(0), I_{34}(0), d_{1}$, and $d_{2}$. Furthermore, from our analysis of the linear mirror, we have available expressions for $I_{12}(0)$ and $I_{34}(0)$ in terms of $\Delta$ and $I_{12}(l)$; these are (4.3) and (4.5), respectively. Thus, if we know $d_{1}, d_{2}$, and $\Delta$, we will be able to solve (4.20) for the reflectivity $I_{12}(l)$. Fortunately, these constants are immediately available to us:

$$
\Delta=\frac{I_{2}(0)+I_{3}(0)-I_{1}(0)-I_{4}(0)}{I_{2}(0)+I_{3}(0)+I_{1}(0)+I_{4}(0)}=\frac{1-M}{1+M}
$$

where we have made use of the boundary conditions (4.20). We observe that $d_{1}$ and $d_{2}$ may be found from the relations $d_{2}-d_{1}=\Delta$ and $d_{1}+d_{2}=1$ so that

$$
d_{1}=M /(M+1)
$$


and

$$
d_{2}=1 /(M+1)
$$

The reflectivity of the ring mirror is shown in Fig. 15 as a function of $\gamma l$. It has been taken to be real so that it represents the $\pi / 2$ phase shift characteristic of holographic recording in photorefractive crystals by diffusion of charge carriers. Curves for several values of the feedback parameter $M$ are included. We see that at threshold, the reflectivity is zero: this threshold may be found by solving the boundary conditions in the limit of zero reflectivity, $I_{12}(n)=0$. It is given by

$$
\gamma l_{t}=\frac{(M+1)}{(M-1)} \ln \left(\frac{M+1}{2 M}\right) \text {. }
$$

Concerning the faithfulness of phase conjugation in the ring mirror, it might be expected that since the self-induced pumping beams are not plane waves a certain amount of distortion would be introduced into the phase conjugate beam. In the case of the linear mirror, physical constraints imposed by the cavity mirrors may lead to filtering of the signal information from the pumping beams, but one of the pumps in the ring mirror is simply light transmitted through the crystal fed back to it by a passive optical system containing, at least in the experiment described below, very little spatial filtering. In the preceding discussion of the linear mirror, we proposed that feedback gives phase conjugate oscillations an advantage in gain over other kinds of oscillation. In the ring mirror we have $A_{4}=M^{1 / 2} K A_{2}$ and $A_{3}^{*}=M^{-1 / 2} K A_{1}^{*}$ with $K$ the lossless linear operator for propagation of beam 2 around the ring to beam 4 . The relevant index grating is represented in the coupled wave equations by a term proportional to $A_{1}^{*} A_{4}+A_{2} A_{3}^{*}$ which equals $M^{1 / 2} A_{1}^{*} K A_{2}+M^{-1 / 2} A_{2} K A_{1}^{*}$ at the crystal face $(z=0)$. Unless $K$ is the identity or otherwise pathological, then both terms in the sum will add in phase at this crystal face if and only if $A_{1}$ is proportional to $A_{2}^{*}$.

The apparatus of Fig. 16 was used to demonstrate the phase conjugating nature of the ring mirror. The expanded and spatially filtered output of an atgon ion laser in single longitude mode at $488.0 \mathrm{~nm}$ passed through lens $L_{2}$ and illuminated an Air Force Resolution Chart. The beam then converged on the passive conjugate mirror, consisting of a single poled crystal of barium titanate in the $M_{1}-M_{2}$ ring cavity. Lens $L_{3}$ was provided to decrease diffractive loss in the ring. The reflected beam was picked off by a beam splitter BS and photographed at the location where a phase conjugate image would be expected. The result is shown in Fig. 17(a). The phase conjugating behavior of the passive phase conjugate mirror is evident although some lack of uniformity in the intensity of image is apparent. This can be seen more clearly in Fig. 17(b), which is the phase conjugate reflection of the uniformly expanded laser beam vignetted by the aperture of lens $L_{2}$. We believe that the dark areas in the image at 6 and 12 o'clock are due to losses via the fanning effect [66] in which a single beam passing through a photorefractive crystal with a sufficiently large coupling loses intensity via holographic two-beam coupling to a broad fan. Fig. 17(c) and (d) shows the effect of fanning on the spatial distribution of intensity in such a signal beam. Mirrors $M_{1}$ and $M_{2}$ were removed from the ring mirror, and holographic gratings in the crystal were allowed to decay by

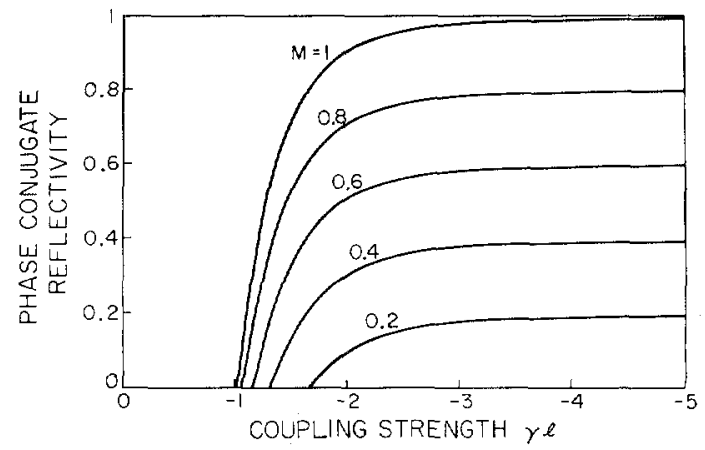

Fig. 15. Reflectivity of the ring passive phase conjugate mirror as a function of coupling strength for several values of the product $M$ of intensity reflectivities of the feedback mirrors $M_{1}$ and $M_{2}$.

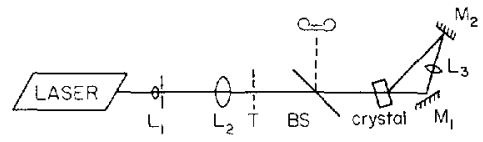

Fig. 16. The experimental arrangement used to demonstrate phase conjugation in the ring passive phase conjugate mirror. An argon ion laser was used at $488 \mathrm{~nm}$ in single longitude mode. Using a Cartesian coordinate system with the abscissa coincident with the beam direction, the locations of the elements measured in $\mathrm{cm}$ were: $30 \times$ objective $L_{1}(61,0)$, transparency $T(30,0)$, beam splitter for observing phase conjugate reflection $B S(5,0) 14 \mathrm{~cm}$ focal length $3 \mathrm{~cm}$ diameter lens $L_{2}(41,0)$, barium titanate crystal $(0,0)$, plane mirror $M_{1},(-9,0)$, plane mirror $M_{2}(-28,-20), 15 \mathrm{~cm}$ focal length $3 \mathrm{~cm}$ diameter lens $L_{3}(-18,-10)$. The $c$-axis of the crystal pointed in the direction of the vector $(0.68,0.73)$. The crystal measured $5.1 \mathrm{X}$ $4.8 \times 5.1 \mathrm{~mm}$ and was poled into a single domain so that the $c$-axis was parallel to the $4.8 \mathrm{~mm}$ side.

dark current leakage. The uniform signal beam was then allowed to pass through the crystal and a photograph [Fig. 17(c)] of this beam after passage through the crystal was immediately taken, before fanning could build up, the time scale of hologram writing being of the order of several seconds. Fig. 17(d) shows the same beam with intensity loss by fanning. A dark area developed, just as dark areas developed in the phase conjugate beam of Fig. 17(b).

\section{The Two Interaction Region Passive Phase Conjugate Mirror}

Recently, Feinberg has reported a passive phase conjugate mirror comprising a single crystal [Fig. 8(d)] which makes use of totally internally reflecting surfaces of the crystal as feedback mirrors [46], [67]. This device is identified as the two interaction region (2IR) mirror since it uses two interaction regions linked inside the single crystal.

By interpreting this device as a ring mirror containing a double phase conjugate mirror in its feedback loop, we can present a simple theoretical analysis making direct use of our previous calculations. We saw above that the transmissivity of the double phase conjugate mirror is the same for both of the beams incident on it. Thus, the 2IR mirror can be thought of as a ring mirror whose feedback $M$ is multiplied by the effective transmission due to the double phase conjugate mirror (4.17). We can immediately write down appropriately modified versions of the ring mirror boundary conditions.

$$
\begin{aligned}
& I_{4}(0) / I_{2}(0)=\mathrm{T}\left(\Delta^{\prime}, a^{\prime}\right) M \\
& I_{1}(0) / I_{3}(0)=\mathrm{T}\left(\Delta^{\prime}, a^{\prime}\right) M
\end{aligned}
$$




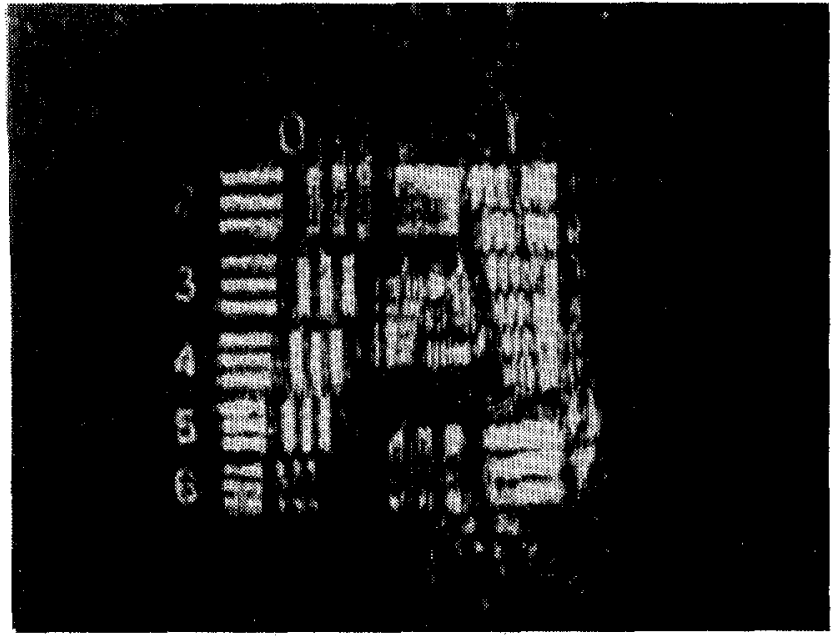

(a)

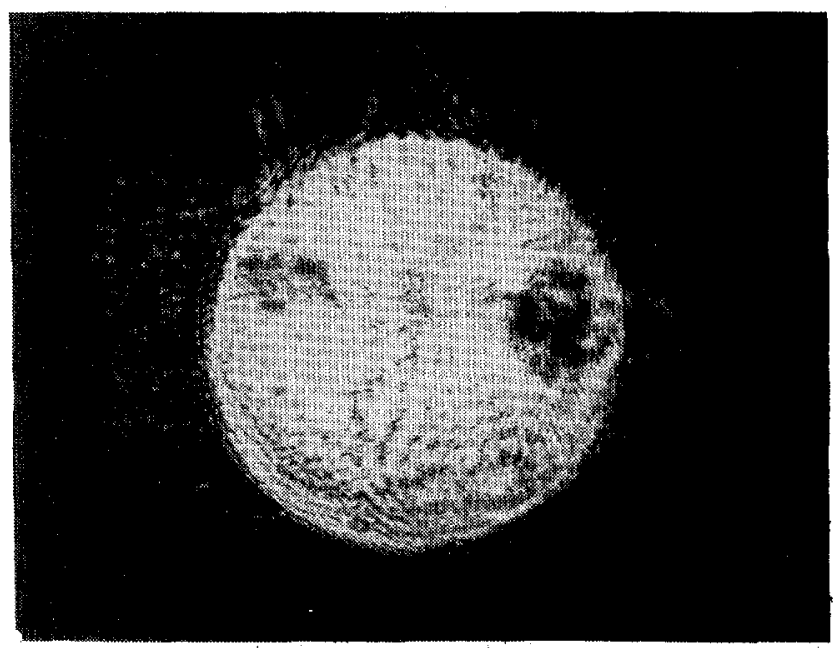

(c)

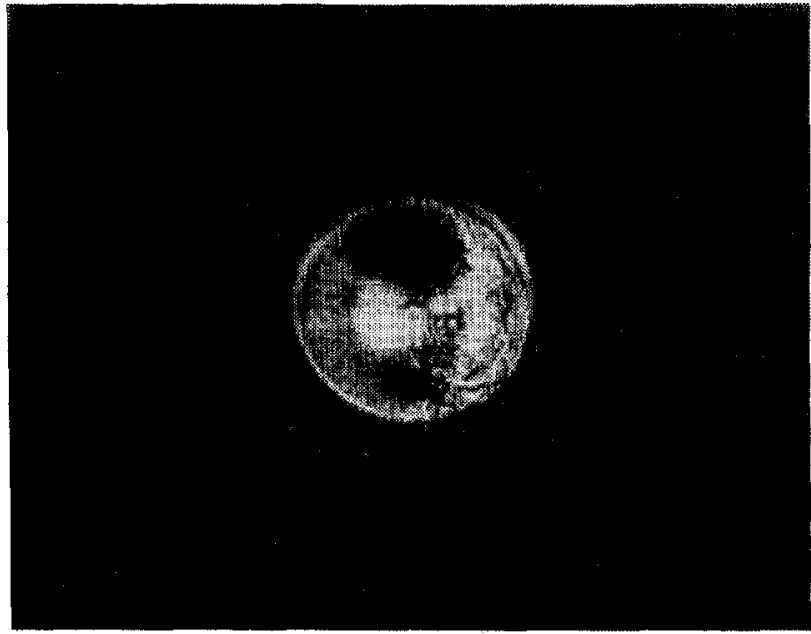

(b)

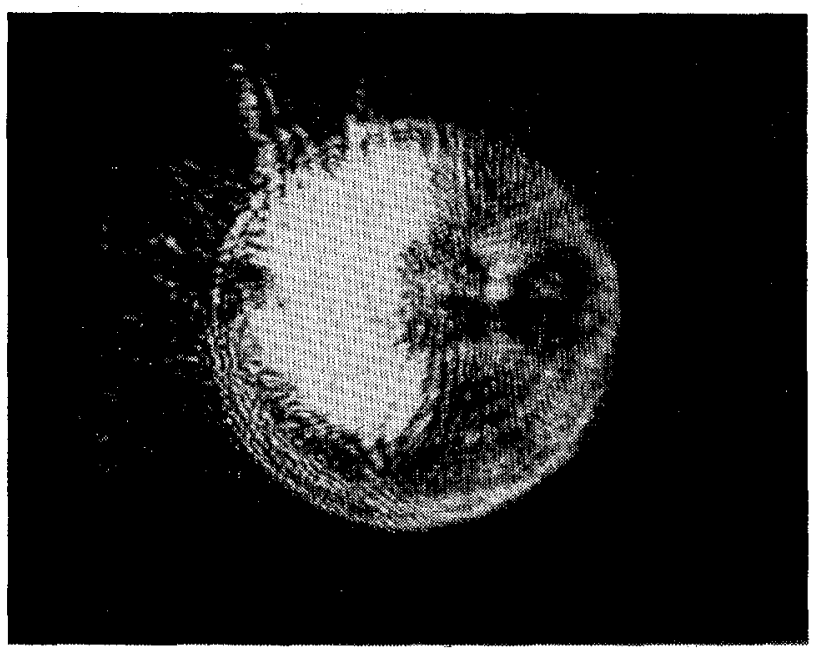

(d)

Fig. 17. Experimental results for the ring mirror showing: (a) The phase conjugate image of an Air Force Resolution Chart. (b) The phase conjugate image of uniform expanded beam vignetted by lens $L_{2}$. (c) The effect of fanning on the signal beam with apparatus as in Fig. 3, without transparency $T$, and mirrors $M_{1}$ and $M_{2}$. Beam transmitted through crystal before buildup of fanning. (d) Beam transmitted through crystal after buildup of fanning.

where primed quantities refer to the double phase conjugate mirror, and the $\Delta$ used in the left-hand side of these equations is given by

$$
\Delta=\frac{1-\mathrm{T}\left(\Delta^{\prime}, a^{\prime}\right) M}{1+\mathrm{T}\left(\Delta^{\prime}, a^{\prime}\right) M} .
$$

These equations (4.24), (4.25) must then be solved numerically for $\Delta^{\prime}$ and reflectivity $R=I_{12}(l)$.

\section{Threshold Versus Reflectivity Versus Ease of Alignment}

Both the linear and ring mirrors have self-starting thresholds $[(4.6)$ and $(4.23)]$ : when they are exceeded, oscillation beams of infinitesimal intensity experience gain. This self-starting ability is not possessed by the semilinear or 2IR mirror since oscillation beams of these devices experience gain only when their intensities are above a certain nonzero threshold. Starting these devices thus requires seeding of their oscillation beams. The 2IR mirror has in fact been shown to be able to start without the aid of externally provided seeding, but it is believed to be dependent on effective seeding by the fanning effect which is due to two-beam coupling amplification of scattered light and not to the four-wave mixing process referred to here.

Equation (4.23) shows that the threshold coupling strength in the ring mirror for unity feedback, $M=1$, is $\gamma l_{t}=-1$. The self-starting threshold for the linear mirror is $\gamma l_{t}=\frac{1}{2} \ln M$ [see (4.6)] where $M$ is the product of the intensity reflectivities of the linear cavity mirrors, so that with ideal feedback $(M=1)$ the threshold coupling strength is zero. The threshold of the semilinear mirror with unity reflectivity for the external mirror is found from (4.13) to be $\gamma l_{t}=-2.49$, while the threshold for the 2IR mirror with ideal feedback is $\gamma l_{t}=-4.68$.

The linear mirror thus has the simultaneous advantages of having very low threshold and of self-starting by four-wave mixing. It does, however, require careful alignment. Of the remaining passive phase conjugate mirrors, all of them easily aligned, the ring mirror seems to be most attractive in that it 


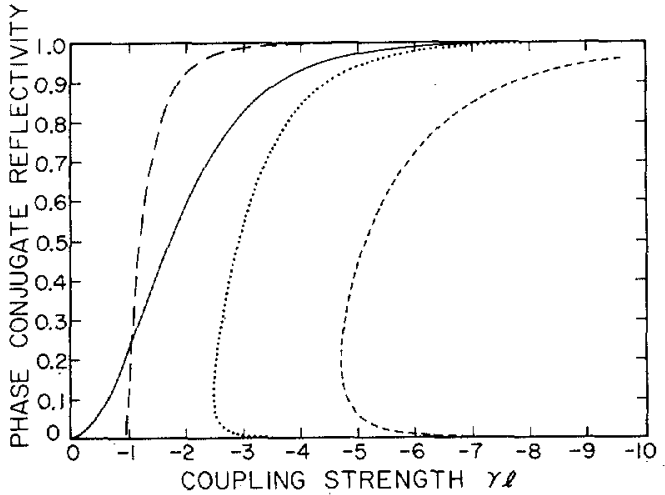

Fig. 18. Graphs of phase conjugate reflectivities of the various passive phase conjugate mirrors with ideal feedback. (a) The linear mirror with $M_{1}=M_{2}=1$, (solid). (b) The semilinear mirror with $M_{2}=1$, (dots). (c) The ring mirror with $M=1$ (long dashes). (d) The two interaction region mirror with $M=1$ (dashes).

has the lowest threshold and is self-starting. A major advantage of the 2IR mirror has been that it is completely selfcontained in a single crystal, using total internal reflection at the crystal faces for feedback. The ring mirror can also be implemented in this same manner using crystals whose surfaces are cut at appropriate angles.

Fig. 18 gives a direct comparison of the reflectivities of each of the PPCM's for ideal feedback in each case. The zero threshold of the linear mirror is shown and we see that the semilinear mirror reflectivity is always lower than that of the full linear mirror, as might be expected, since with one mirror removed, there is always going to be some extra loss. The ring mirror reflectivity, after taking off from its threshold, soon exceeds all of them.

\section{CONCLUSION}

The two vital elements in this work have been the solution of the coupled wave equations with pump depletion and the availability of PR crystals with large coupling strengths. The value of such concurrent theoretical and experimental work is undeniable: it has led to many new useful devices such as passive and double phase conjugate mirrors and their demonstration in aberration correction in lasers and image processing. In the future we can expect similarly important developments, and as research proceeds into optimizing the photorefractive effect, the applications of these devices to problems in image processing and aberration correction are bound to multiply.

\section{ACKNOWLEDGMENT}

M. Cronin-Golomb would like to acknowledge the support of the University of Sydney and B. Fischer would like to acknowledge the support of the Weizmann post-doctoral fellowship. The authors would like to acknowledge helpful discussions with S.-K. Kwong.

\section{REFERENCES}

[1] A. Ashkin, G. D. Boyd, J. M. Dziedzic, R. G. Smith, A. A. Ballman, J. J. Levinstein, and K. Nassau, "Optically-induced refractive index inhomogeneities in $\mathrm{LiNbO}_{3}$ and $\mathrm{LiTaO}_{3}, "$ Appl. Phys. Lett., vol. 9, pp. 72-74, 1966.

[2] F. S. Chen, "A laser-induced inhomogeneity of refractive indices in KTN," J. Appl. Phys., vol. 38, pp. 3418-3420, 1967.

[3] - "Optically induced change of refractive indices in $\mathrm{LiNbO}_{3}$ and $\mathrm{LiTaO}_{3}$,"J. Appl. Phys., vol. 40, pp. 3389-3396, 1969.
[4] F. S. Chen, J. T. LaMacchia, and D. B. Fraser, "Holographic storage in lithium niobate," Appl. Phys. Lett., vol. 13, pp. 223225,1968 .

[5] R. L. Townsend and J. T. LaMacchia, "Optically induced refractive index changes in $\mathrm{BaTiO}_{3}, "$ J. Appl. Phys., vol. 41, pp. 5188$5192,1970$.

[6] J. B. Thaxter and M. Kestigian, "Unique properties of SBN and their use in a layered optical memory," Appl. Opt., vol. 13, pp. 913-924, 1974.

[7] A. M. Glass, D. von der Linde, and T. J. Negran, "High-voltage bulk photovoltaic effect and the photorefractive process in $\mathrm{LiNbO}_{3}, "$ Appl. Phys. Lett., vol. 25, pp. 233-235, 1974.

[8] J. P. Huignard, J. P. Herriau, and F. Micheron, "Optical storage in $\mathrm{LiNbO}_{3}: \mathrm{Fe}$ with selective erasure capability," Rev. Phys. Appl., vol. 10, pp. 417-423, 1975.

[9] D. von der Linde and A. M. Glass, "Photorefractive effects for reversible holographic storage of information," Appl. Phys., vol. 8, pp. 85-100, 1975.

[10] J.P. Huignard and F. Micheron, "High sensitivity read-write volume holographic storage in $\mathrm{Bi}_{12} \mathrm{SiO}_{20}$ and $\mathrm{Bi}_{12} \mathrm{GeO}_{20}$ crystals," Appl. Phys. Lett., vol. 29, pp. 591-593, 1976.

[11] K. Megumi, H. Kozuka, M. Kobayashi, and Y. Furuhita, "Highsensitive holographic storage in Ce-doped SBN," Appl. Phys. Lett., vol. 30, pp. 631-633, 1977.

[12] R. Orlowski, E. Krätzig, and H. Kurz, "Photorefractive effects in $\mathrm{LiNbO}_{3}: \mathrm{Fe}$ under external electric fields," Opt. Commun., vol. 20, pp. 171-174, 1977 .

[13] J.P. Huignard and J.P. Herriau, "Real-time double exposure interferometry with $\mathrm{Bi}_{12} \mathrm{SiO}_{20}$ crystals in transverse electrooptic configuration," Appl. Opt., vol. 16, pp. 1807-1809, 1977.

[14] M. Peltier and F. Micheron, "Volume hologram recording and charge transfer process in $\mathrm{Bi}_{12} \mathrm{SiO}_{20}$ and $\mathrm{Bi}_{12} \mathrm{GeO}_{20}$," J. Appl. Phys., vol. 48, pp. 3683-3690, 1977.

[15] J. P. Huignard, J.P. Herriau, and T. Valentin, "Time average holographic interferometry with photoconductive electrooptic $\mathrm{Bi}_{12} \mathrm{SiO}_{20}$ crystals," Appl. Opt., vol. 16, pp. 2796-2798, 1977.

[16] H. Kurz, "Lithium niobates as a material for holographic information storage," Philips Tech Rev., vol. 37, pp. 109-120, 1977.

[17] J. P. Herriau, J. P. Huignard, and P. Aubourg, "Some polarization properties of volume holograms in $\mathrm{Bi}_{12} \mathrm{SiO}_{20}$ cyrstals and applications," Appl. Opt., vol. 17, pp. 1851-1853, 1978.

[18] M. P. Petrov, S. I. Stepanov, and A. A. Kamshilin, "Holographic storage of information and peculiarities of light diffraction in birefringent electrooptic crystals," Opt. Laser Tech., pp. 149151, June 1979.

[19] R. Orlowski, L. A. Boatner, and E. Krätzig, "Photorefractive effects in the cubic phase of potassium tantalate-niobate," Opt. Commun, vol. 35 , pp. $45-48,1980$.

[20] J. O. White and A. Yariv, "Real time image processing via fourwave mixing in a photorefractive medium," Appl. Phys. Lett., vol. 37, pp. 5-7, 1980.

[21] J. P. Huignard and A. Marrakchi, "Coherent signal beam amplification in two-wave mixing experiments with photorefractive $\mathrm{Bi}_{12} \mathrm{SiO}_{20}$ crystals," Opt. Commun., vol. 38, pp. 249-254, 1981.

[22] J. J. Amodei, "Analysis of transport processes during holographic recording in insulators," RCA Rev., vol. 32, pp. 185-198, 1971.

[23] Y. Ninomaya, "Recording characteristics of volume holograms," J. Opt. Soc. Amer., vol. 63, pp. 1124-1130, 1973.

[24] L. Young, W.K.Y. Wong, M.L.W. Thewalt, and W. D. Cornish, "Theory of formation of phase holograms in lithium niobate," Appl. Phys. Lett., vol, 24, pp. 264-265, 1974.

[25] D. M. Kim, R, R. Shah, T. A. Rabson, and F. K. Tittel, "Nonlinear dynamic theory for photorefractive phase hologram formation," Appl. Phys. Lett., vol. 28, pp. 338-340, 1975.

[26] D. W. Vahey, "A nonlinear coupled-wave theory of holographic storage in ferroelectric materials," J. Appl. Phys., vol. 46, pp. $3510-3515,1975$.

[27] S. F. Su and T. K. Gaylord, "Unified approach to the formation of phase holograms in ferroelectric crystals," $J$. Appl. Phys., vol. 46, pp. 5208-5213, 1975.

[28] R. Magnusson and T. K. Gaylord, "Use of dynamic theory to describe experimental results for volume holography," J. Appl. Phys., vol. 47, pp. 190-199, 1976.

[29] M. G. Moraham and L. Young, "Hologram writing by the photorefractive effect," J. Appl. Phys., vol. 48, pp. 3230-3236, 1977.

[30] B. I. Sturman, "Interaction of two light waves in a crystal caused by photoelectron diffusion and drift," Sov. Phys.-Tech. Phys., vol. 23, pp. 589-595, 1978. 
[31] N. V. Kukhtarev, V. B. Markov, S. G. Odulov, M. S. Soskin, and V. L. Vinetskii, "Holographic storage in electrooptic crystals," Ferroelectrics, vol. 22, pp. 949-960, 1979.

[32] J. Feinberg, D. Heiman, A. R. Tanguay, Jr., and R. W. Hellwarth, "Photorefractive effects and light induced charge migration in barium titanate," J. Appl. Phys., vol. 51, pp. 1297-1305, 1980.

[33] J. Feinberg, "Real-time edge enhancement using the photorefractive effect," Opt. Lett., vol. 5, pp. 330-332, 1980.

[34] S. I. Stepanov, V. V. Kulikov, and M. P. Petrov, "Running holograms in photorefractive $\mathrm{Bi}_{12} \mathrm{SiO}_{20}$ crystals," Opt. Commun., vol. 44, pp. $19-23,1982$

[35] For reviews see A. Yariv, "Phase conjugate optics and real-time holography," IEEE J. Quantum Electron., vol. QE-14, pp. 650660,1978 , also Special issue on Optical Phase Conjugation, Opt. Electron., vol. 21, no. 2, also Optical Phase Conjugation, R. A. Fisher, Ed. New York: Academic, 1983.

[36] A. Yariv, "Four wave nonlinear optical mixing as real time holography," Opt. Commun., vol. 25, pp. 23-25, 1978.

[37] J. P. Huignard, J. P. Herriau, P. Aubourg, and E. Spitz, "Phase conjugate wavetront generation via real time holography in $\mathrm{Bi}_{12}$ $\mathrm{SiO}_{20}$ crystals," Opt. Lett., vol. 4, pp. 21-23, 1979.

[38] J. P. Huignard, J. P. Herriau, and G. Rivet, "Phase conjugate and spatial frequency dependence of wavefront reflectivity in $\mathrm{Bi}_{12}$ $\mathrm{SiO}_{20}$ crystals," Opt. Lett., vol. 5, pp. 102-104, 1980.

[39] N. V. Kukhtarev, "Wavefront reversal of optical beams in anisotropic media," Sov. J. Quantum Electron., vol. 11, pp. 878-883, 1981.

[40] P. N. Gunter, "Electric field dependence of phase-conjugate wavefront reflectivity in reduced $\mathrm{KNbO}_{3}$ and $\mathrm{Bi}_{12} \mathrm{GeO}_{20}$," Opt. Lett., vol. $7, \mathrm{pp} .10-12,1982$.

[41] J. Feinberg, "Phase conjugating mirror with continuous wave gain," Opt. Lett., vol. 5, pp. 519-521, 1980.

[42] J. O. White, M. Cronin-Golomb, B. Fischer, and A. Yariv, "Coherent oscillation by self-induced gratings in the photorefractive crystal $\mathrm{BaTiO}_{3}, "$ Appl. Phys. Lett., vol. 40, pp. 450-452, 1982.

[43] M. Cronin-Golomb, B. Fischer, J. Nilsen, J. O. White, and A. Yariv, "Laser with dynamic holographic intracavity distortion correction capability," Appl. Phys. Lett., vol. 41, pp. 219-220, 1982.

[44] M. Cronin-Golomb, B. Fischer, J. O. White, and A. Yariv, "Passive (self-pumped) phase conjugate mirror: Theoretical and experimental investigation," Appl. Phys. Lett., vol. 41, pp. 689691,1982

[45] - "A passive phase conjugate mirror based on self-induced oscillation in an optical ring cavity," Appl. Phys. Lett., vol. 42, pp. 919-921, 1983.

[46] J. Feinberg, "Self-pumped, continuous-wave phase conjugator using internal reflection," Opt. Lett., vol. 7, pp. 486-488, 1982.

[47] S. G. Odulov and M. S. Soskin, "Laser with degenerate pumping on $\mathrm{LiNbO}_{3}$,"JETP Lett., vol. 37, pp. 289-293, 1983.

[48] B. Fischer, M. Cronin-Golomb, J. O. White, A. Yariv, and R. Neurgaonkar, "Amplifying continuous wave phase conjugate mirror with strontium barium niobate," Appl. Phys. Lett., vol. 40 , pp. 863-865, 1982.

[49] P. Gunter, "Holography, coherent light amplification and optical phase conjugation with photorefractive materials," Phys. Rep., vol. 93, pp. 199-299, 1982.

[50] A. Yariv, Quantum Electronics. New York: Wiley, 1975.

[51] B. Fischer, M. Cronin-Golomb, J. O. White, and A. Yariv, "Amplified reflection, transmission and oscillation in real-time holography," Opt. Lett., vol. 6, pp. 519-521, 1981.

[52] A. Yariv and D. M. Pepper, "Amplified reflection, phase conjugation, and oscillation in degenerate four wave mixing," Opt. Lett., vol. 1, pp. $16-18,1977$

[53] J. F. Lam, "Spectral response of nearly degenerate four-wave mixing in photorefractive materials," Appl. Phys. Lett., vol. 42, pp. 155 -157, 1983.

[54] J. H. Marburger and J. F. Lam, "Nonlinear theory of degenerate four-wave mixing," Appl. Phys. Lett., vol. 34, pp. 389-391, 1979.

[55] - "Effects of nonlinear index changes on degenerate fourwave mixing," Appl. Phys. Lett., vol. 35, pp. 249-251, 1979.

[56] H. G. Winful and J. H. Marburger, "Hysteresis and optical bistability in degenerate four-wave mixing," Appl. Phys. Lett., vol. 36 , pp. 613-614, 1980 .

[57] A. R. Kessel and V.M. Musin, "Exact solution of shortened equations of non-linear parametric processes," Opt. Commun., vol. 44, pp. 133-134, 1982.

[58] M. Cronin-Golomb, B. Fischer, J. O. White, and A. Yariv, "Exact solution of a nonlinear model of four-wave mixing and phase conjugation," Opt. Lett., vol. 7, pp. 313-315, 1982.

[59] J. Au Yeung, D. Fekete, D. Pepper, and A. Yariv, "A theoretical and experimental investigation of the modes of optical resonators with phase conjugate mirrors," IEEE J. Quantum Electron., vol. QE-15,pp. 1180-1188, 1979.

[60] I. M. Bel"yugdin, M. G. Galushkin, and E. M. Zemskov, "Properties of resonators with wavefront-reversing mirrors," Sov. J. Quantum Electron., vol. 9, pp. 20-23, 1979.

[61] I. M. Bel'yugdin and E. M. Zemskov, "Theory of resonators with wavefront reversing mirrors," Sov. J. Quantum Electron., vol. 9, pp. 1198-1199, 1979

[62] P. A. Bélanger, A. Hardy, and A. E. Siegman, "Resonant modes of optical cavities with phase-conjugate mirrors," Appl. Opt., vol. 19, pp. $602-609,1980$.

[63] J. F. Lam and W. P. Brown, "Optical resonators with phase-conjugate mirrors," Opt. Lett., vol, 5, pp. 61-63, 1980.

[64] M. G. Reznikov and A. I. Khizhnyak, "Properties of a resonator with a wavefront-reversing mirror," Sov. J. Quantum Electron., vol. 10, pp. 633-634, 1980 .

[65] R. C. Lind and D. G. Steel, "Demonstration of the longitude modes and aberration-correction properties of a continuous wave dye laser with a phase conjugate mirror," Opt. Lett., vol. 6, pp. $554-556,1981$.

[66] V. V. Voronov, I. R. Dorosh, Yu. S. Kus'minov, and N. V. Tkachenko, "Photoinduced light scattering in cerium-doped barium strontium niobates crystals," Sov. J. Quantum Electron., vol. 10, pp. $1346-1349,1980$

[67] K. R. MacDonald and J. Feinberg, "Theory of self-pumped phase conjugator with two coupled interaction regions," J. Opt. Soc. Amer., vol. 73, pp. 548-553, 1983.

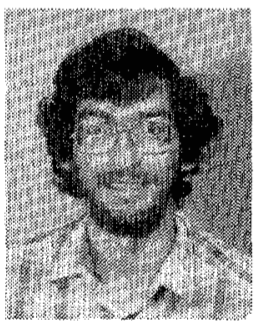

Mark Cronin-Grolomb received the B.Sc. degree in physics from the University of Sydney, Sydney, Australia, in 1979, and the Ph.D. degree in physics from the California Institute of Technology, Pasadena, in 1983.

As an undergraduate, he investigated the use of diffraction gratings in enhancing the characteristics of solar selective film absorbers. Since 1979 he has been at the California Institute of Technology, working first on a neutrino scattering experiment at the Fermi National Accelerator Laboratory, and then carrying out research in nonlinear optics and phase conjugation in photorefractive materials. He is currently a Research Fellow in Applied Physics.

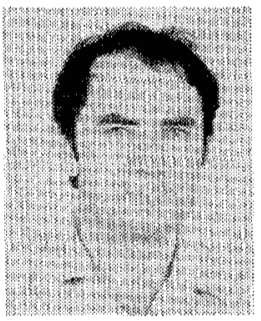

Baruch Fischer received the Ph.D. degree in physics from Bar Ilan University, Israel, in 1980.

From 1981 to 1982 he was a Weizmann Postdoctoral Research Fellow at the California Institute of Technology and is now a Senior Lecturer at the Department of Electrical Engineering, Technion, Haifa, Israel. His current research interests are electrooptics and quantum electronics.

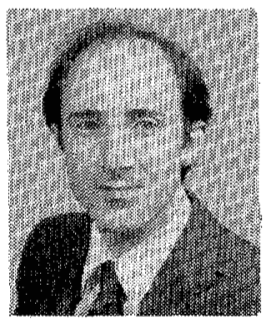

Jeffrey $\mathbf{O}$. White was born in New Jersey. He received the $\mathrm{Sc} . \mathrm{B}$. degree in physics with honors from Brown University, Providence, RI, in 1977. He received the Ph.D. degree in applied physics from the California Institute of Technology, Pasadena.

His research interests include phase conjugation, the photorefractive effect, optical information transmission and processing, chaotic behavior in nonlinear optics, compensation of distortions using nonlinear optics, self-pumped 
phase conjugate mirrors (patent pending), and solving coupled nonlinear partial differential equations. He is coauthor of a chapter in the text Optical Phase Conjugation.

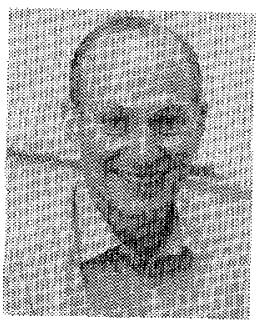

Amnon Yariv (S'56-M'59-F'70) was born in Tel Aviv, Israel, on April 13, 1930. He received the B.S., M.S., and Ph.D. degrees, all in electrical engineering, from the University of California, Berkeley, in 1954, 1956, and 1958, respectively. He was with Bell Laboratories, Murray Hill, $\mathrm{NJ}$, in 1959, during the early stages of the laser effort. He joined the California Institute of Technology in 1964 as an Associate Professor of Electrical Engineering and became a Professor in 1966. In 1980 he became the Thomas G. Myers Professor of Electrical Engineering and Applied Physics. He has taken part (with various co-workers), in the discovery of a number of early solid-state laser systems, in the formulation of the theory of parametric quantum noise and the prediction of parametric fluorescence, in the invention of the technique of mode-locked uttrashort-pulse lasers and FM lasers, in the introduction of GaAs and CdTe as infrared electrooptic and window materials, in proposing and demonstrating semiconductor-based integrated optics technology, and in pioneering the field of phase conjugate optics. His present research efforts are in the areas of nonlinear optics, recombination mechanisms in semiconductors, and semiconductor lasers and integrated optics, especially the problem of monolithic integration of transistors, injection lasers, and detectors for high frequency applications. He has authored or coauthored some 300 papers in professional journals, as well as a number of basic texts in quantum electronics, optics, and quantum mechanics. He is also an Associate Editor of Optics Communications and the Journal of Applied Physics, and was previously Associate Editor of the IEEE JournaL of Quantum Electronics. He is a consultant to the Hughes Aircraft Research Laboratories and is a founder and Chairman-of-the-Board of ORTEL, Inc.

Dr. Yariv is a member of the American Physical Society, Phi Beta Kappa, and the National Academy of Engineering, and a Fellow of the Optical Society of America. He is the recipient of the 1980 Quantum Electronics Award of the IEEE.

\title{
Nonlinear Optical Interfaces: Switching Behavior
}

\author{
PETER W. SMITH, FELLOW, IEEE, AND W. J. TOMLINSON
}

\begin{abstract}
We report the results of a study of the switching behavior of the interface between an ordinary dielectric and a nonlinear dielectric material with an intensity-dependent index of refraction. The nonlinear dielectric used for these experiments consisted of a liquid suspension of dielectric particles. Because this medium has a very large effective nonlinearity, it was possible to perform these experiments with the $\mathrm{CW}$ output of an argon ion laser. Our experimental results are in excellent agreement with the predictions of numerical calculations based on a two-dimensional Gaussian input beam. We also report the observation of an unstable reflectivity state with a lifetime $\sim 10^{3}$ times the response time of the nonlinear medium.
\end{abstract}

\section{INTRODUCTION}

$I^{\mathrm{N}}$ $\mathrm{N}$ this paper we report on a study of the switching behavior of a nonlinear interface [1]-[4] (an interface between an ordinary dielectric and a dielectric material with an intensitydependent index of refraction). For the experiments, we have made use of an artificial Kerr medium, consisting of a liquid suspension of dielectric particles [5]- [7]. This medium has a very large effective nonlinear refractive index, which makes it possible to study the behavior of nonlinear interfaces with moderate-intensity $\mathrm{CW}$ laser beams. The experimental results are in qualitative and in some cases quantitative agreement with the results of numerical calculations for two-dimensional Gaussian beams [4], and they exhibit features that are not predicted by simple plane-wave theories. No evidence of a bistable reflectivity was obtained. We did observe, however, an unstable state with a lifetime as long as $10^{3}$ times the response time of

Manuscript received July 8, 1983.

The authors are with Bell Laboratories, Holmdel, NJ 07733. the nonlinear medium. This long-lived state may have serious implications for some proposed applications of nonlinear interfaces, and it merits further study.

Nonlinear interfaces are of interest because they are potentially useful as very fast (sub-ps) optical switches and logic elements. The initial theoretical study of nonlinear interfaces, assuming an incident plane wave, predicted that for the configuration we are studying, the reflectivity of the interface is unity at low intensities, and for intensities above a threshold value the reflectivity will switch to a lower value [1]. The plane-wave theory also predicted that for a finite range of intensities below the threshold value there is a second stable state, with a reflectivity of less than unity, so that the reflectivity is bistable. The first experiments on such an interface gave results that appeared to be consistent with the predictions of the plane-wave theory [2], [3]. However, the apparatus used for these experiments had several features which complicated the interpretation of the results. The input light beam had a Gaussian profile, so it was not possible to make direct comparisons between the predictions of the plane-wave theory and the experimental results. The input pulses were $\sim 1 \mathrm{~ns}$ long, and the detection system had a time response of $\sim 300 \mathrm{ps}$, thus averaging over any rapid changes in reflectivity. Finally, since the experiments were done for only a single input pulse width, it was not possible to distinguish between the observed apparent bistable reflectivity, and an unstable state with a lifetime longer than the input pulse duration.

In an attempt to obtain theoretical predictions for Gaussian profile input beams, several authors have carried out steadystate numerical simulations for two-dimensional Gaussian 